The Astrophysical Journal Supplement Series, 63:615-644, 1987 March

(1) 1987. The American Astronomical Society. All rights reserved. Printed in U.S.A.

\title{
CONTINUUM ENERGY DISTRIBUTIONS OF QUASARS IN THE PALOMAR-GREEN SURVEY
}

\author{
G. Neugebauer \\ Palomar Observatory, California Institute of Technology \\ R. F. GREEN \\ Kitt Peak National Observatory, National Optical Astronomy Observatories ${ }^{1}$ \\ AND \\ K. Matthews, M. Schmidt, B. T. Soifer, And J. Bennett \\ Palomar Observatory, California Institute of Technology \\ Received 1986 May 20; accepted 1986 August 25
}

\begin{abstract}
Nearly simultaneous observations, from 0.3 to $2.2 \mu \mathrm{m}$, of the complete set of quasars found in the Palomar-Green survey are presented, along with 3.7 and $10.1 \mu \mathrm{m}$ observations of a limited number of the quasars. The majority of the continua at rest frequencies between $10^{13.8}$ and $10^{15.3} \mathrm{~Hz}$ are consistent with fits by two power-law slopes plus a " $3000 \AA$ bump." The spectral indices representing the lower frequency observations have a median value of -1.4 , while those describing the higher frequencies have a median value of -0.2 . The transition frequency between these slopes is close to $10^{14.5} \mathrm{~Hz}$ in the rest frame; the dispersion around this frequency is about one octave. A significant fraction of the low-resdshift quasars show evidence of nebulosity within $5^{\prime \prime}$ of the quasar.
\end{abstract}

Subject headings: quasars - spectrophotometry

\section{INTRODUCTION}

The Palomar-Green (PG) survey of stellar objects with ultraviolet excess (Green, Schmidt, and Liebert 1986) covered an area of 10,714 square degrees at Galactic latitudes above $30^{\circ}$ and has resulted in the identification of a large number of bright quasars. Schmidt and Green (1983) have identified 114 of the objects in the PG survey as quasars and bright Seyfert galaxies. This subset, the Palomar bright quasar survey (BQS), is described in detail by Schmidt and Green, and consists of all objects in the PG catalog which fulfill the morphological criterion that they have a dominant starlike appearance on blue prints of the $1.2 \mathrm{~m}$ Palomar Observatory Schmidt Sky Atlas and the spectroscopic criterion that they show broad emission lines with substantial redshift. The BQS is complete to an average limiting $B$-magnitude of $16.16 \mathrm{mag}$.

In this paper, continuum energy distributions are given, from $0.32 \mu \mathrm{m}$ to either $2.2,3.7$, or $10.1 \mu \mathrm{m}$, of 105 quasars in the PG sample; all but seven are in the BQS. The intent has been to obtain nearly simultaneous visual and infrared observations of an unbiased and complete selection of quasars. The results extend and complement the study of the continua of quasars by Neugebauer et al. (1979, hereafter NOBM). The observations form one of the bases for a series of papers (e.g., Bechtold et al. 1987; Elvis et al. 1986) in which the survey or individual objects are discussed and interpreted.

\footnotetext{
${ }^{1}$ Operated by the Association of Universities for Research in Astronomy, Inc., under contract with the National Science Foundation.
}

\section{OBSERVATIONS}

The visual observations were made at the Cassegrain focus of the Hale $5 \mathrm{~m}$ telescope with the multichannel spectrophotometer (Oke 1969). In all cases a focal-plane diaphragm of $15^{\prime \prime}$ diameter was used with decker settings allowing $0.008 \mu \mathrm{m}$ bandpasses for wavelengths from 0.32 to $0.57 \mu \mathrm{m}$ and 0.016 $\mu \mathrm{m}$ bandpasses from 0.57 to $0.79 \mu \mathrm{m}$. The channels sensitive at wavelengths from 0.8 to $1.0 \mu \mathrm{m}$ did not add useful information because of the low number of total counts recorded, and these observations are not further considered. Absolute fluxes were derived for each night using a flux standard sdG star on the AB79 system (Oke and Gunn 1983); standard extinction corrections appropriate for Palomar were applied. The statistical uncertainties in the observations were typically $2 \%$, while the uncertainty in the absolute calibration is assumed also to be $2 \%$.

Near-infrared observations were also made at the Cassegrain focus of the Hale $5 \mathrm{~m}$ telescope. For wavelengths between 1.3 and $3.7 \mu \mathrm{m}$, observations were made in the standard photometric bands with an InSb photovoltaic detector with a 5".5 diameter focal-plane diaphragm; observations at $10.1 \mu \mathrm{m}$ were made using a Ge bolometer with a 4".5 diameter focal-plane diaphragm. Sky emission was canceled by subtracting the sky $15^{\prime \prime}$ to the north and south of the object. The infrared photometry for wavelengths between 1.3 and $3.7 \mu \mathrm{m}$ was calibrated with respect to the standard stars given in Elias et al. (1982); the photometry at $10.1 \mu \mathrm{m}$ was calibrated with respect to standard stars which are effectively on the system of Tokunaga (1984). The photometric uncer- 
TABLE 1

Absolute Calibration

\begin{tabular}{cc|cc}
\hline \hline $\begin{array}{c}\text { Wavelength } \\
(\mu \mathrm{m})\end{array}$ & $\begin{array}{c}f_{\nu}(0 \mathrm{mag}) \\
(\mathrm{Jy})\end{array}$ & $\begin{array}{c}\text { Wavelength } \\
(\mu \mathrm{m})\end{array}$ & $\begin{array}{c}f_{\nu}(0 \mathrm{mag}) \\
(\mathrm{Jy})\end{array}$ \\
\hline $0.55 \ldots \ldots \ldots$ & 3530 & $2.20 \ldots \ldots$ & 646 \\
$1.27 \ldots \ldots \ldots$ & 1580 & $3.7 \ldots \ldots$. & 250 \\
$1.65 \ldots \ldots \ldots$ & 1040 & $10.1 \ldots \ldots$ & 37 \\
\hline
\end{tabular}

tainties are typically less than $5 \%$ at $1.3-2.2 \mu \mathrm{m}, 10 \%$ at 3.7 $\mu \mathrm{m}$, and $20 \%$ at $10.1 \mu \mathrm{m}$. Magnitudes were converted to flux densities using the flux density values for $0.0 \mathrm{mag}$ given in Table 1 . The uncertainties in the adopted infrared absolute calibration are typically $<5 \%$, and this system ties smoothly into the multichannel AB79 calibration.

The multichannel and 1.3, 1.6, and $2.2 \mu \mathrm{m}$ observations presented here were made during 1980 unless otherwise indicated. Observations of a single object in the visual and in the infrared to $2.2 \mu \mathrm{m}$ were generally made within a period of two months in order to minimize the effects of variability. The majority of the infrared observations were repeated in 1986 to obtain a second epoch of measurements in order to study the potential variability of these objects. These measurements are being prepared for separate publication.

The flux densities $f_{\nu}$ from 0.32 to $2.2 \mu \mathrm{m}$ obtained within a few months of each other are tabulated in Table 2 as a function of the frequency $\nu$ in the observer's frame for 105 quasars. The quoted uncertainties include the statistical and absolute uncertainties mentioned above. Two quasars were measured at nonstandard wavelengths with the multichannel spectrometer. The flux densities for these are given in Table 2B.

Seven of the 105 objects listed in Table 2 are not included in the BQS. Except that the blue photographic magnitude was fainter than the limiting magnitude of the BQS sample, six of these seven satisfied all criteria of the $\mathrm{BQS}$. The six were included in the present study but were omitted from the BQS because the observing lists for the present study were made up from the PG sample before the completeness criteria were finally applied to yield the BQS. The seventh source, PG $2349-014$, was omitted from the BQS because the object was measured in error to have a photographic $U-B$ color redder than the selection limit of the BQS.

For 16 sources included in the BQS, observations are not included in Table 2. Seven of these have subsequently been identified as low-luminosity Seyfert galaxies, three were added to the $\mathrm{BQS}$ sample after the present observations were made, and one is within $3^{\prime \prime}$ of a star of comparable magnitude. Three more quasars, PG 0051+124 (I Zw 1), PG 1425+267 (Ton 202), and PG 2308+089 (4C 09.72) were not observed as part of this program, but observations of all three have been previously published (Oke and Lauer 1979; NOBM; Richstone and Schmidt 1980). Two quasars in the BQS were not observed in both the visible and near-infrared because of observing time limitations.

The continuum energy distributions of the 105 quasars are plotted in Figure 1 as a function of the rest frequencies $\nu_{0}$.
Several corrections have been applied to the raw observations to produce the flux densities shown in Figure 1 and given in Table 2. The principal correction was the removal of spectral lines from the measurements in order to obtain continuum energy distributions free of contamination from emission lines. Other corrections include those to account for extinction in the Galaxy and an adjustment to account for 3.7 and $10.1 \mu \mathrm{m}$ measurements which were not made close in time to the visible and 1.3-2.2 $\mu \mathrm{m}$ observations.

For the multichannel observations, a set of pseudocontinuum intervals was adopted to avoid the strongest emission features while leaving a grid of sampling points spaced throughout the $0.32-0.8 \mu \mathrm{m}$ region. The prescription used in defining these "continuum intervals" was to classify known emission features as strong, moderate, or weak, and then to choose continuum intervals that lie outside specified velocity widths of the line peaks. For strong lines-Lyman- $\alpha, C$ Iv, C III], $\mathrm{Mg}$ II, and $\mathrm{H} \alpha$ - the velocity width was chosen to be $10,000 \mathrm{~km} \mathrm{~s}^{-1}$. For moderate-strength lines- $\mathrm{O}$ VI, $\mathrm{N} \mathrm{v}$, $\mathrm{Si}$ IV-O IV], $\mathrm{H} \gamma, \mathrm{H} \beta$, [O III], and the red $\mathrm{He}$ I lines-a velocity width of $5000 \mathrm{~km} \mathrm{~s}^{-1}$ was adopted. The higher order Balmer series and other known forbidden lines were classified as weak lines and assumed to have a velocity width of 2500 $\mathrm{km} \mathrm{s}^{-1}$. The wavelength interval in the rest frame from 0.290 to $0.385 \mu \mathrm{m}$ was left intact for the exercise of fitting the " 3000 $\AA$ bump," as discussed below. The final continuum intervals that were adopted are presented in Table 3 .

The ubiquity of $\mathrm{Fe}$ II emission presents a major problem in estimating the continuum energy distribution of the quasars. Although the strong multiplets are explicitly avoided, the large number of lines may raise the entire measured continuum above the "true" underlying continuum (see Wills, Netzer, and Wills 1985, hereafter WNW); the strength of the iron line contribution to the blue continuum could range from less than $10 \%$ to being the dominant component (Wampler 1985 ). Since the continuum determined from points with rest wavelengths longer than $0.5 \mu \mathrm{m}$ and shorter than $0.2 \mu \mathrm{m}$ should not be strongly affected in most objects, the Fe II uncertainties will be reflected mostly in the determination of the strength of the " $3000 \AA$ bump."

For the higher redshifts in the sample, the $\mathrm{H} \alpha, \mathrm{H} \beta$, and $O$ III lines can be redshifted into the bandpasses of the near-infrared continuum filters. The effects of these lines were removed from the infrared continuum measurements by assuming rest-frame equivalent widths of $0.040,0.007$, and $0.004 \mu \mathrm{m}$, respectively, for the three lines, and velocity widths of $9000 \mathrm{~km} \mathrm{~s}^{-1}$ for the hydrogen lines and $900 \mathrm{~km} \mathrm{~s}^{-1}$ for the oxygen lines. The corrections to the observed flux densities never exceeded 25\%, and were larger than 5\% for only 16 of the 105 quasars.

The effect of Galactic extinction for each quasar was removed using the reddening deduced by Burstein and Heiles (1984) for the area near the quasar in question. The corrections in the flux densities were then found by applying the van de Hulst extinction curve No. 15 (van de Hulst 1946; see Rieke and Lebofsky 1985).

Observations of 48 objects at $3.7 \mu \mathrm{m}$ and of 39 objects at $10.1 \mu \mathrm{m}$ were obtained of the 105 sources. These measure- 


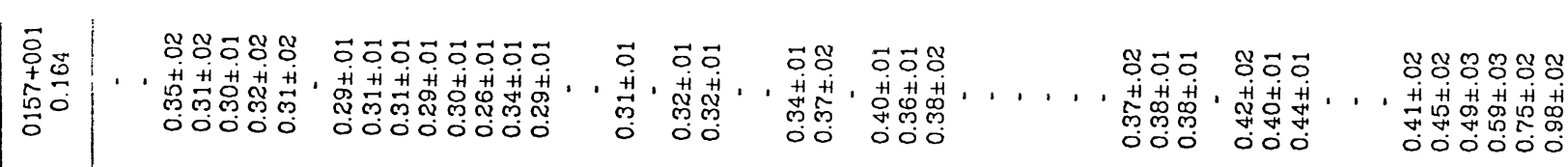

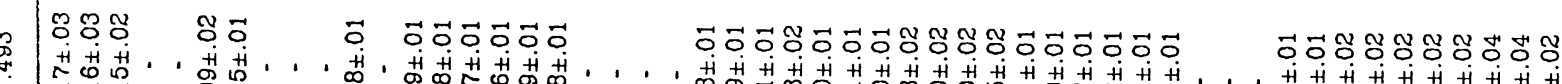

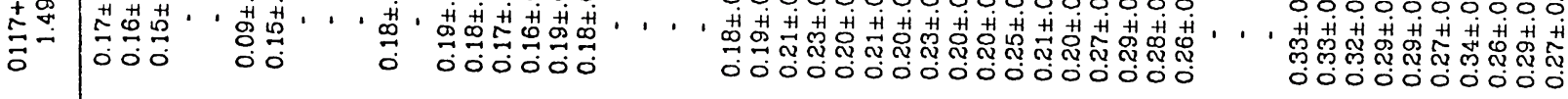

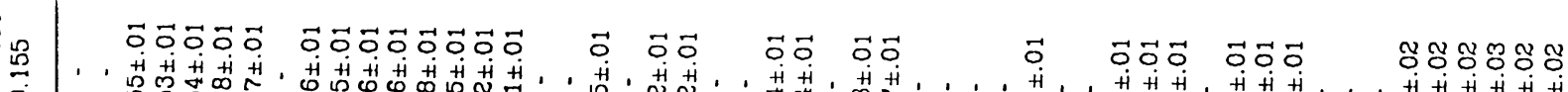

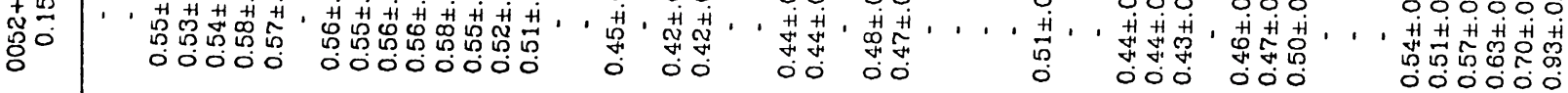

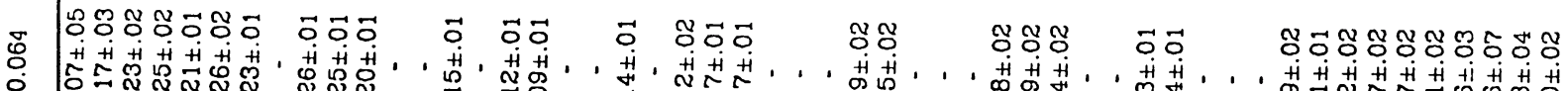

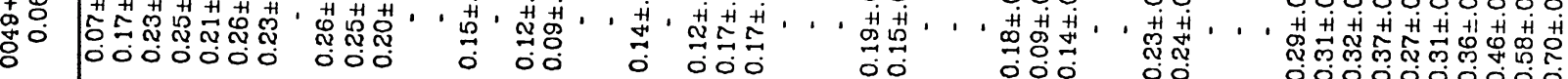

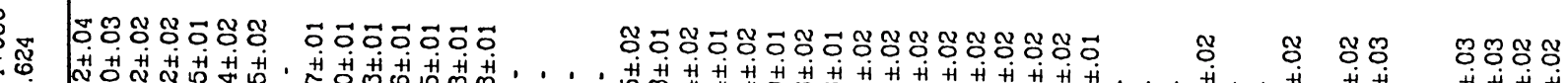

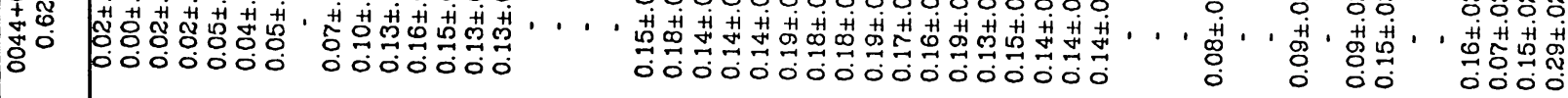

N ᄀ

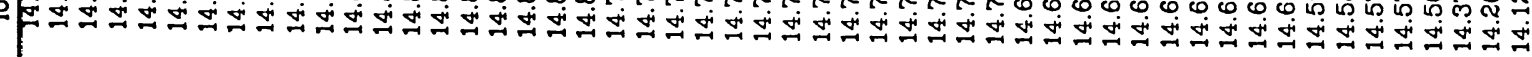

ßु)

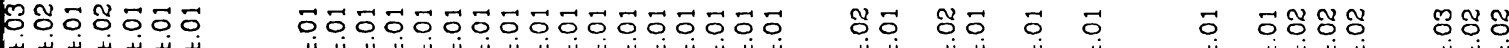

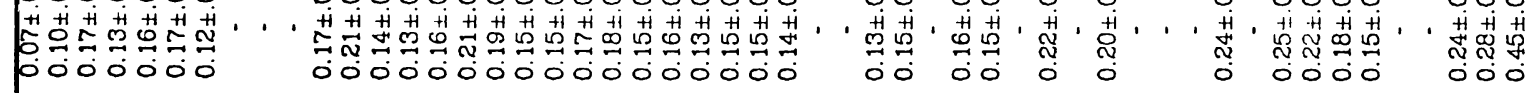

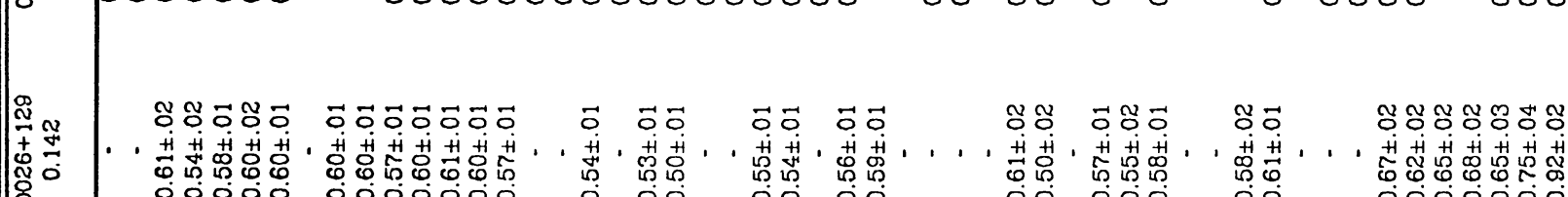

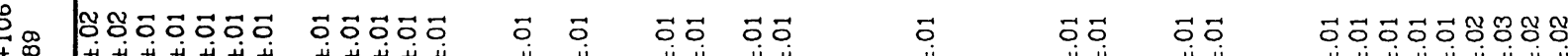

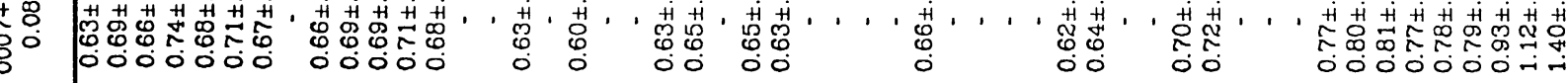

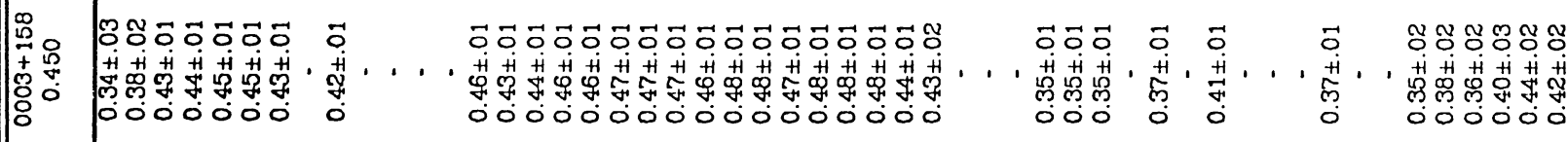

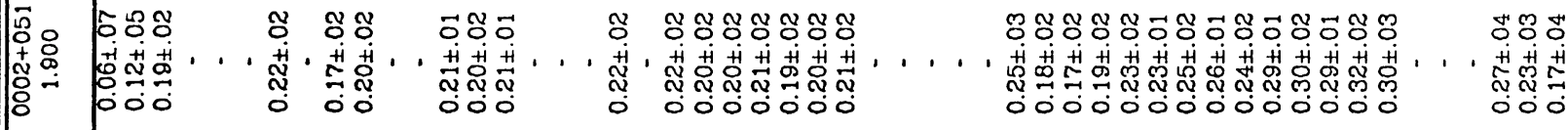

热 |مी 


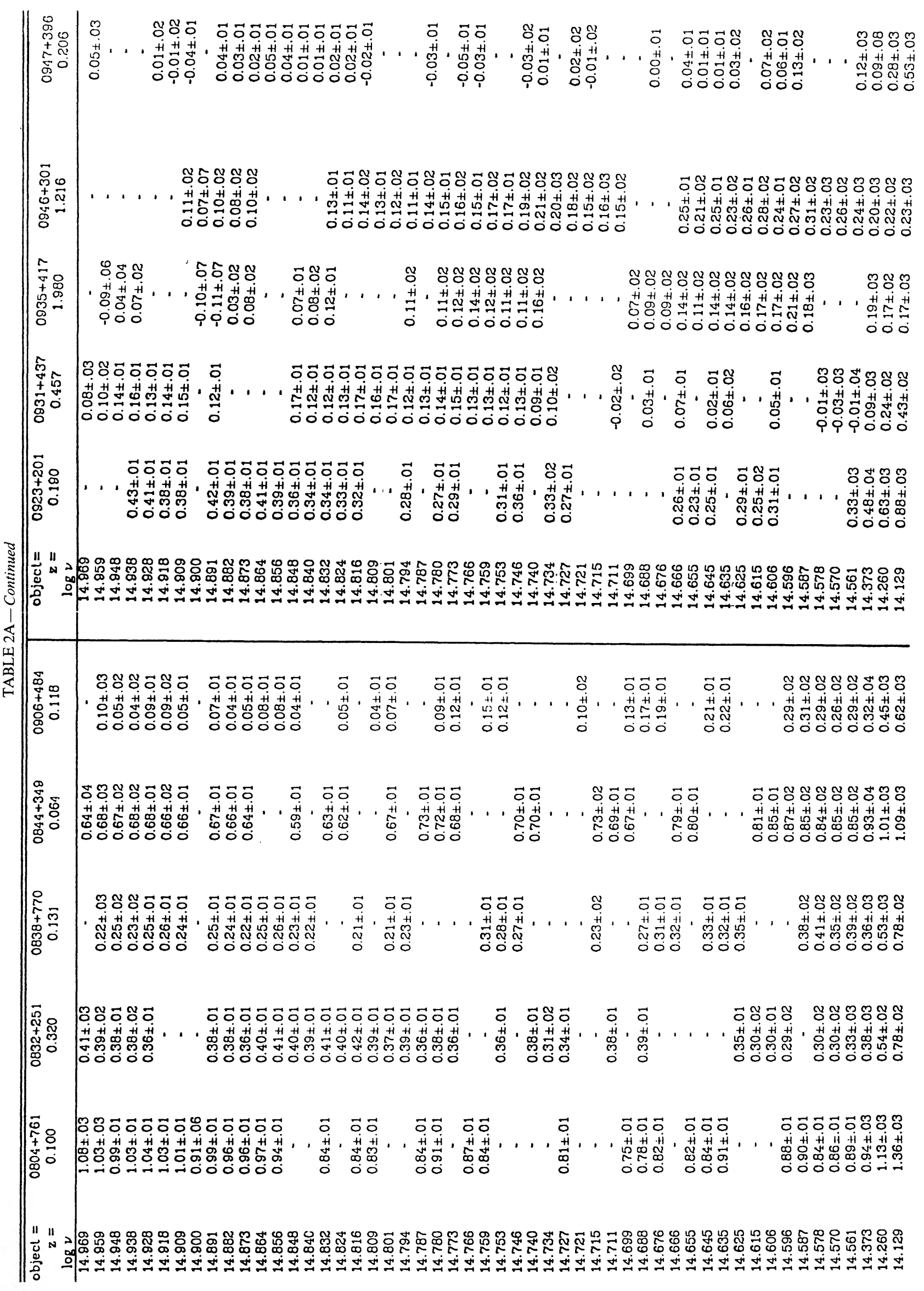




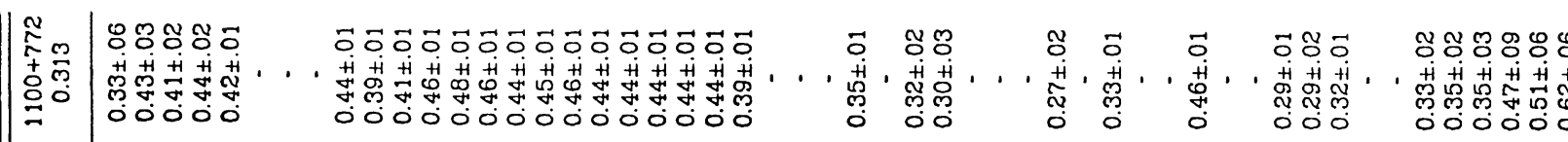

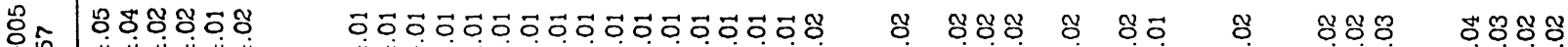
多怘

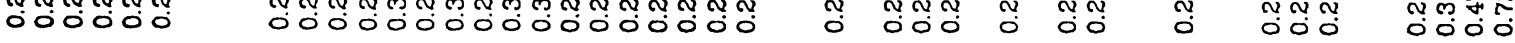

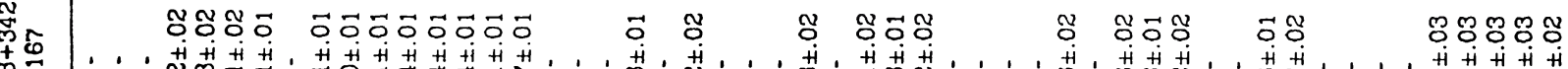

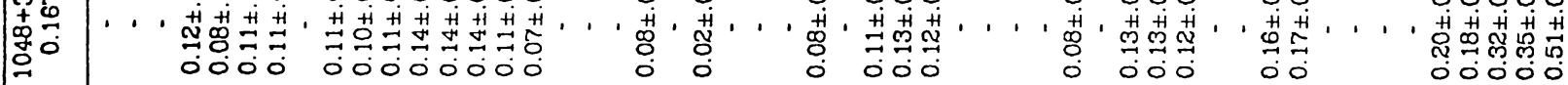

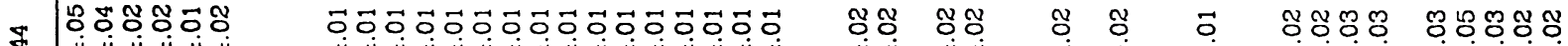

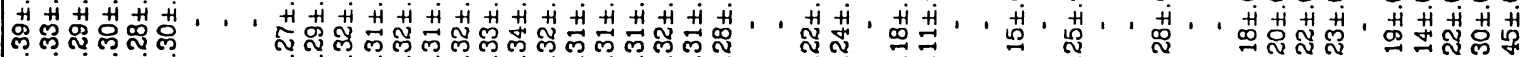
$000000 \quad 0000000000000000$ 00 00 0 0 o 000000000

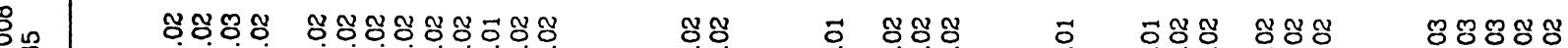

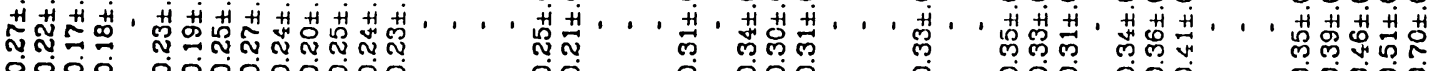
0000000000000 00 0 000 0 000 000

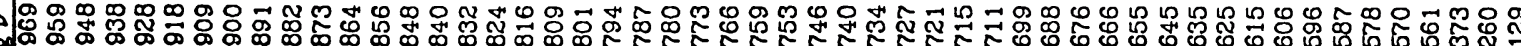

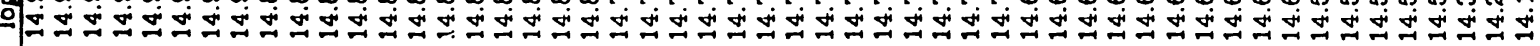

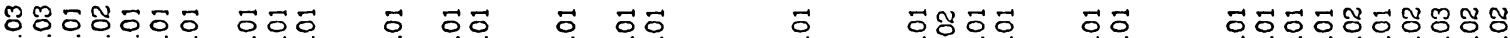

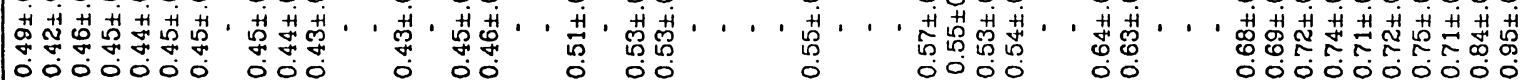

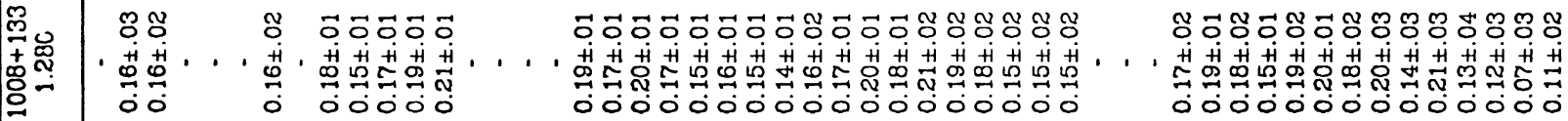

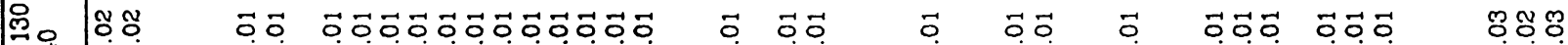

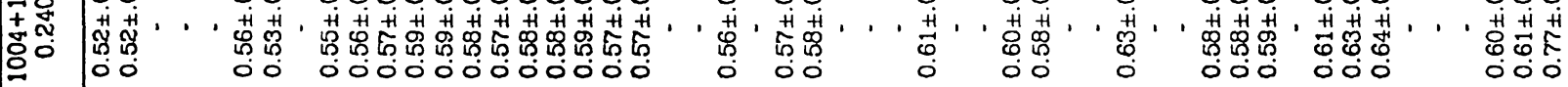

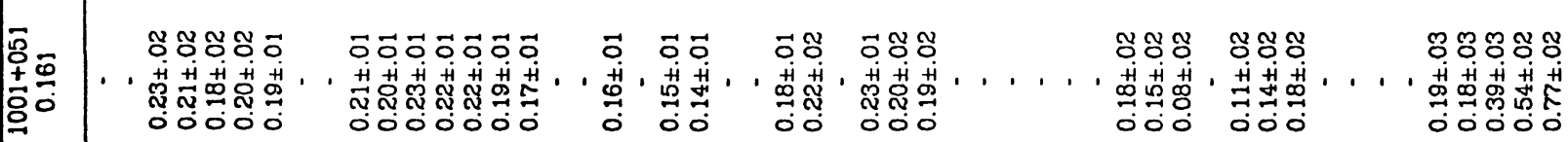

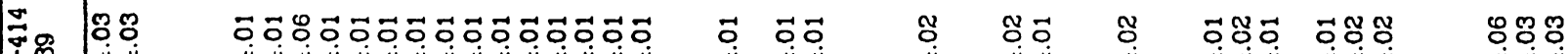

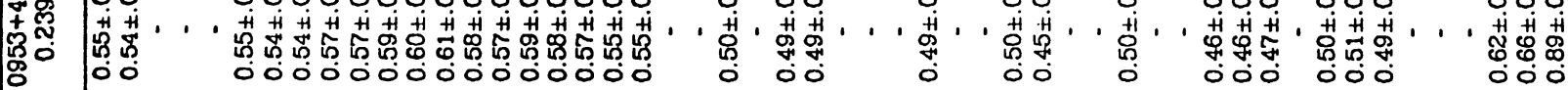

"I " 워 


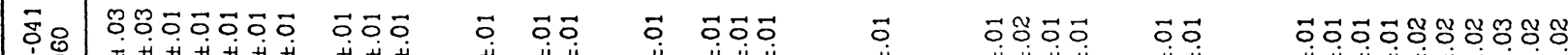

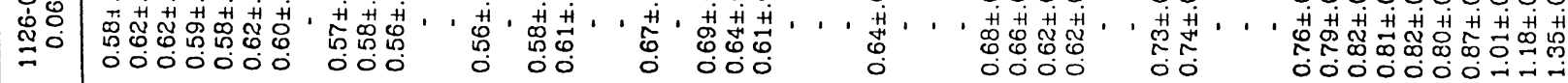

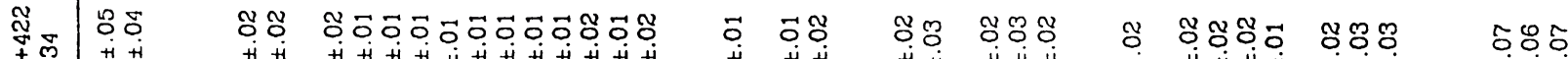

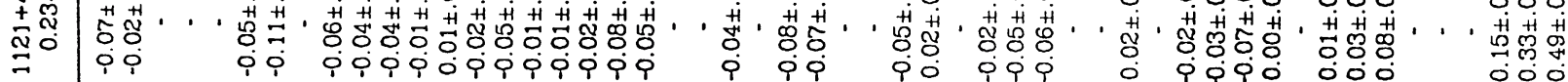
(

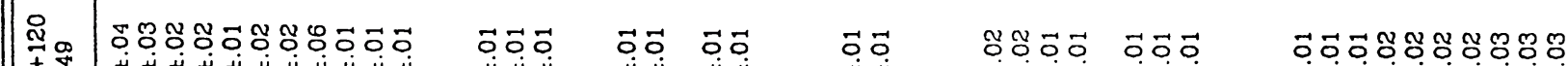

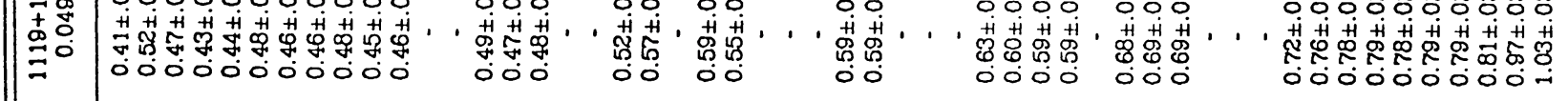

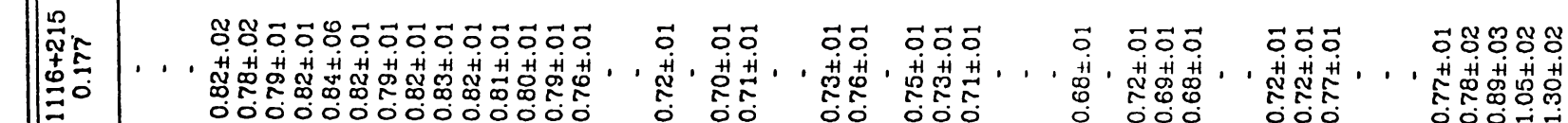
00000000000000
0

" "

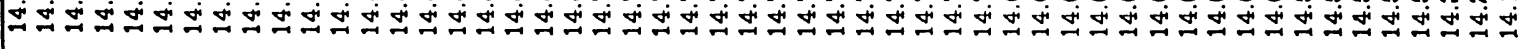

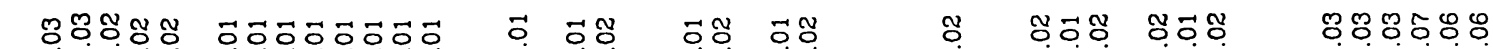

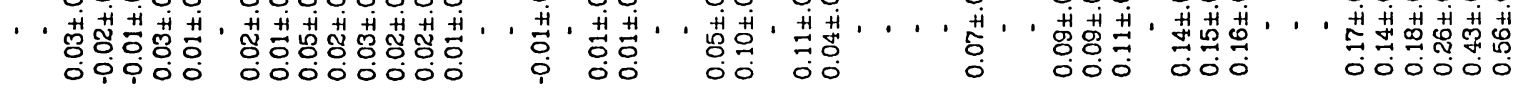
i 00

$$
000000
$$

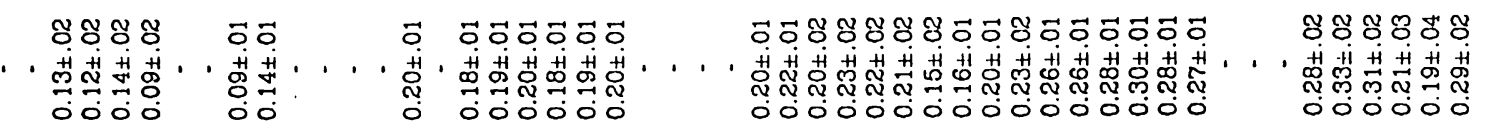

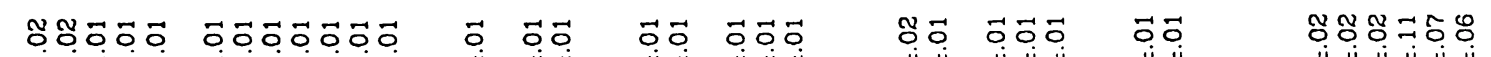

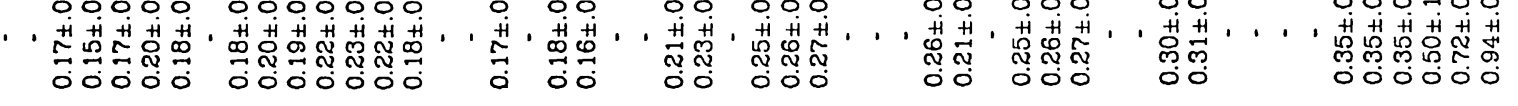

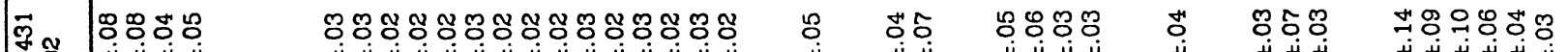

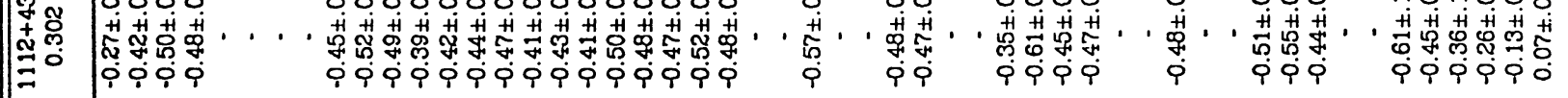
|

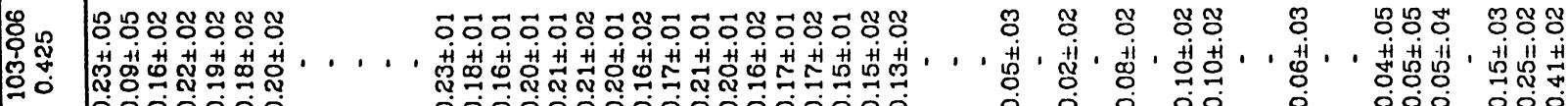

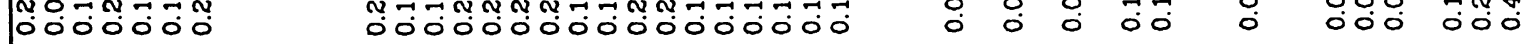

" "

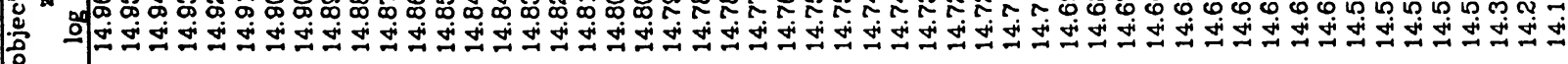




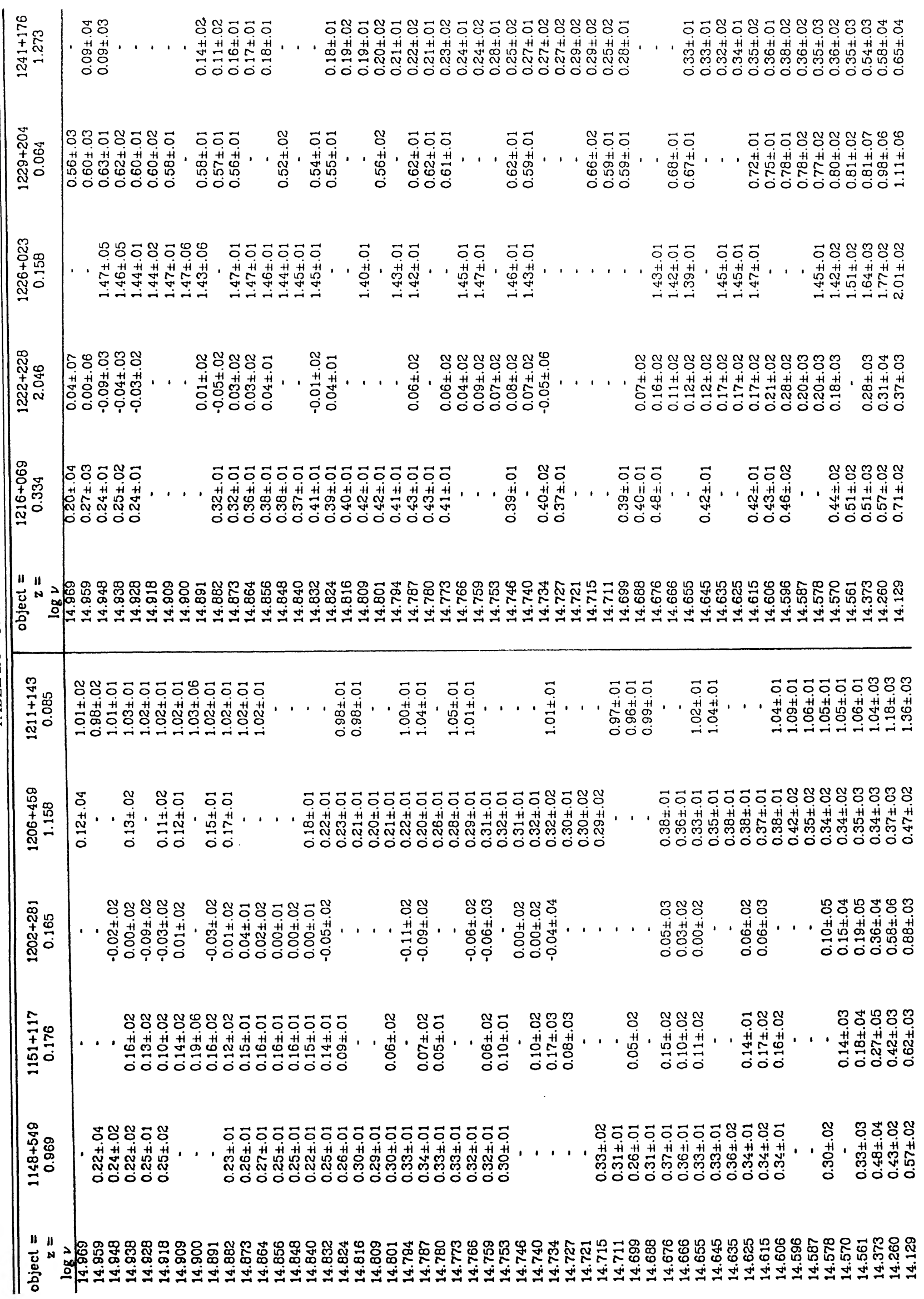




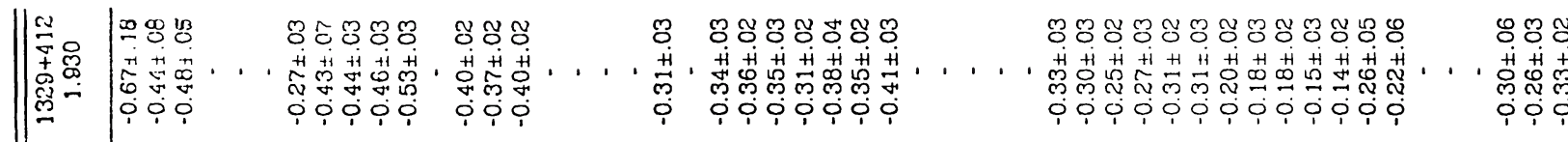

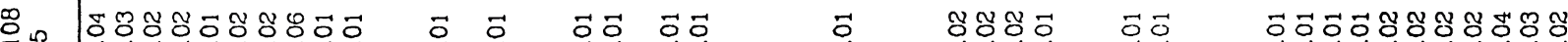

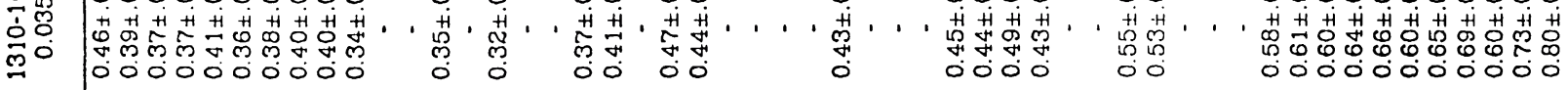

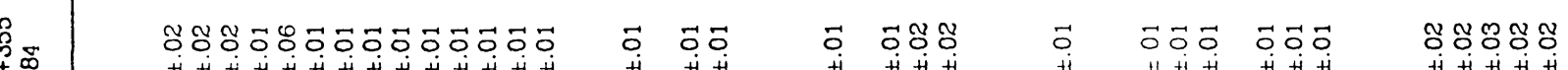

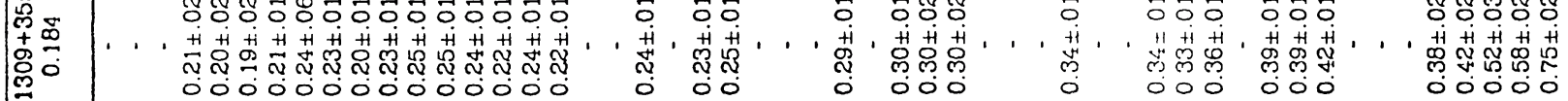

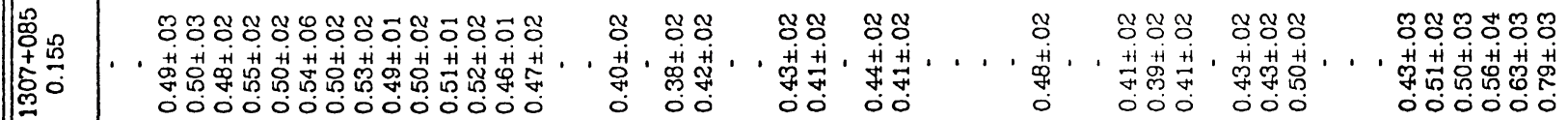

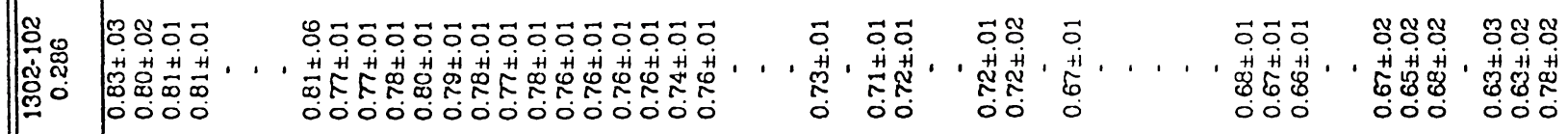

" " ఎ

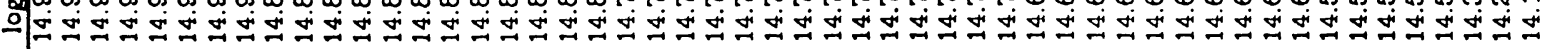

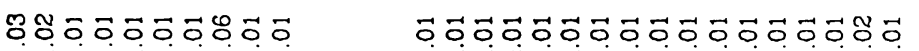

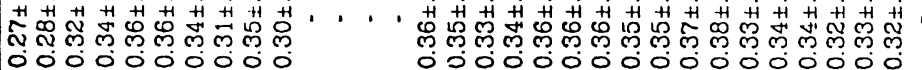

$\overrightarrow{0} \quad \overrightarrow{0} \overrightarrow{0}$

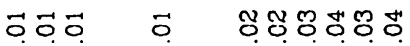

0000000

ஸे

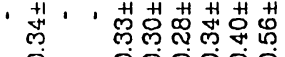

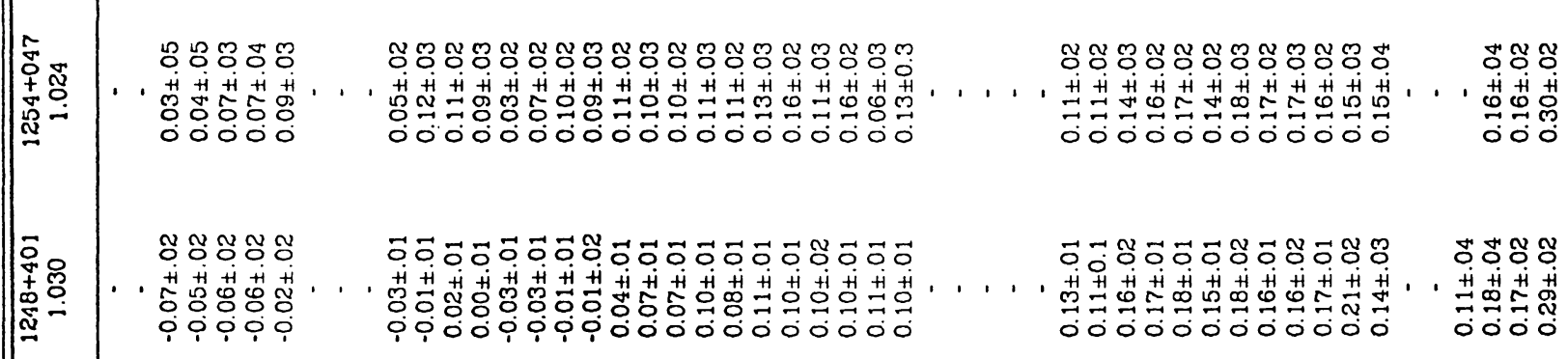

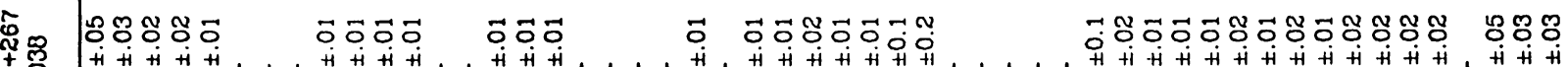

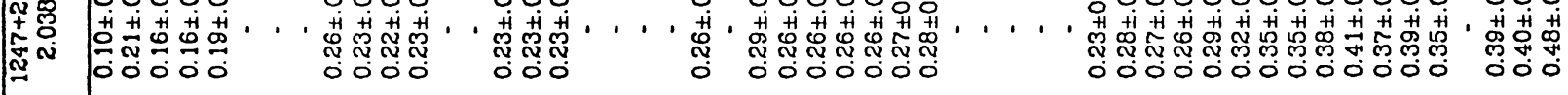

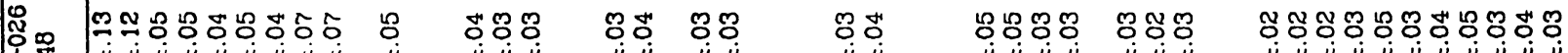

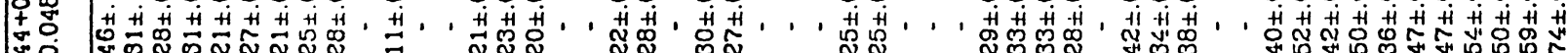

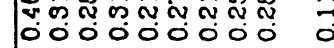

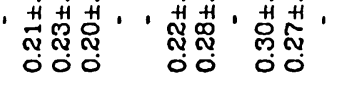
00 0000 Ho

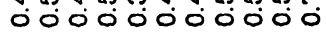

" “

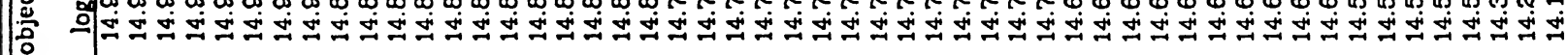




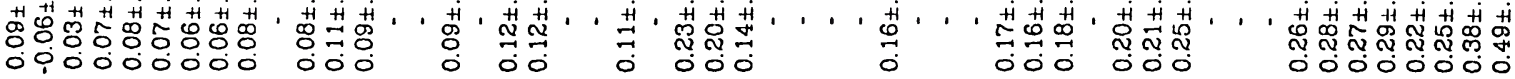

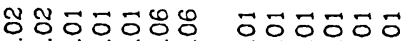

\section{$\overrightarrow{0} \overrightarrow{0} \overrightarrow{0} \quad \overrightarrow{0} \overrightarrow{0} \overrightarrow{0} \tilde{0}$}

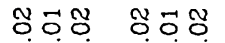

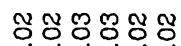

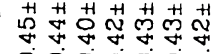

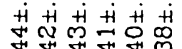

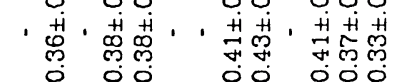

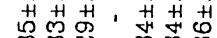

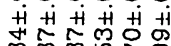

मूण

000 ०००

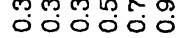

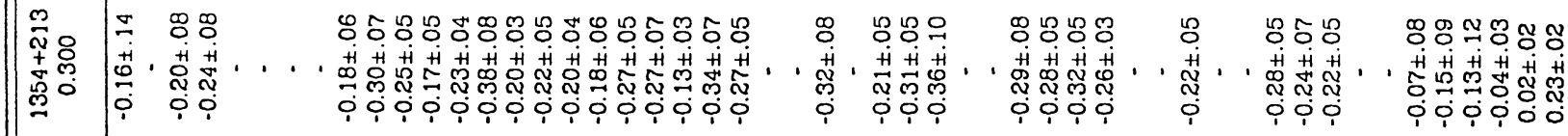

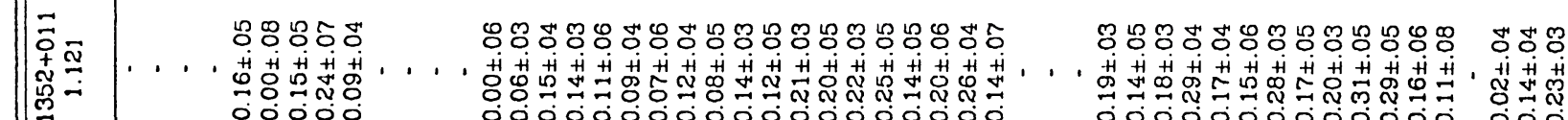

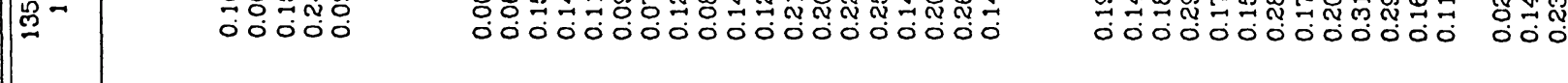

"

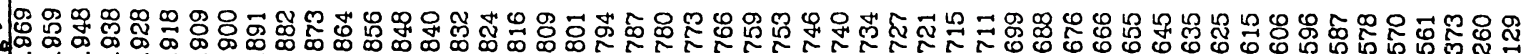

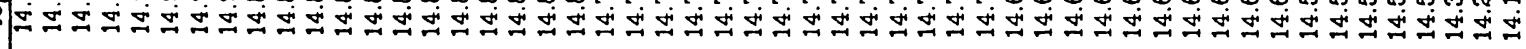

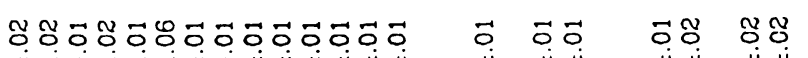

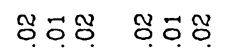

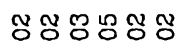

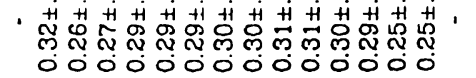

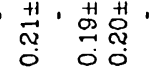

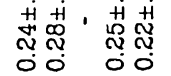

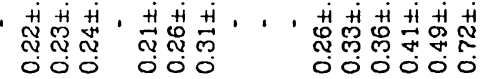

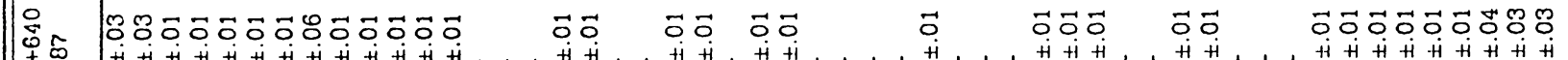

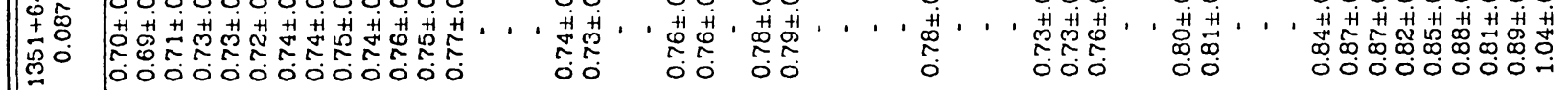

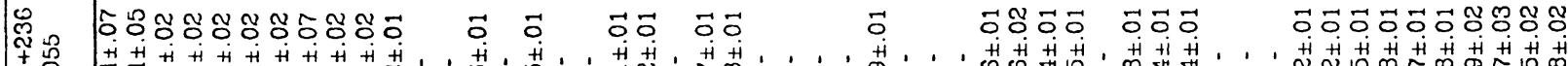

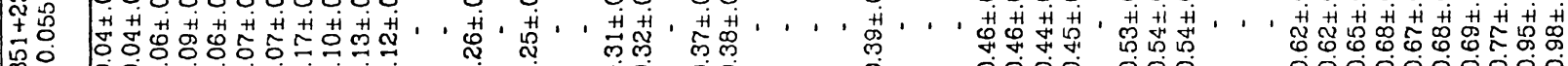

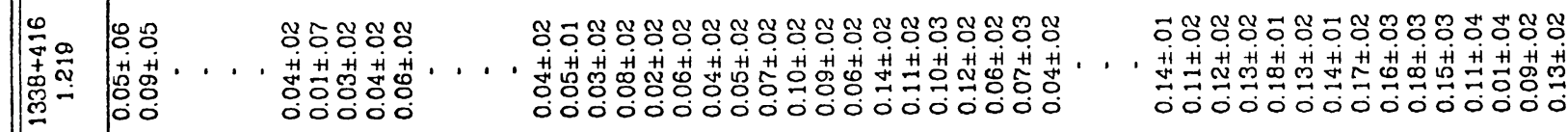

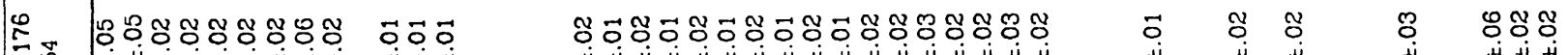

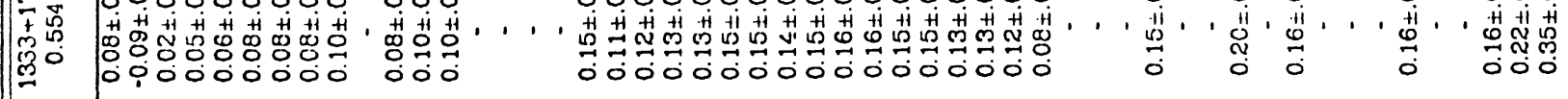

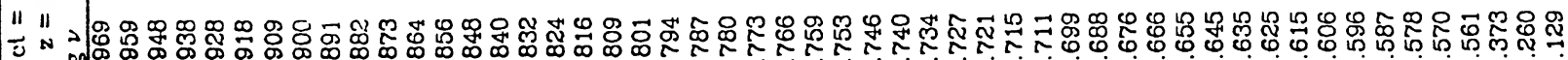
党 


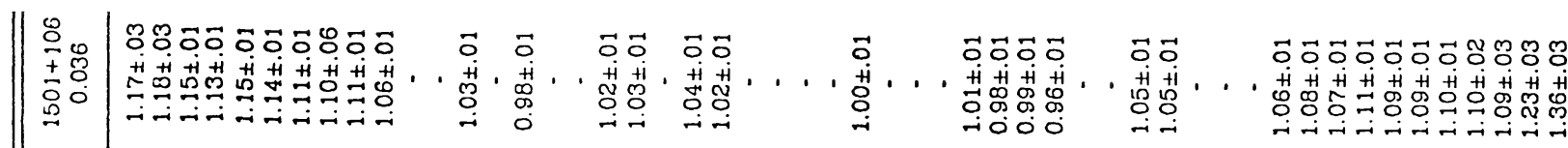

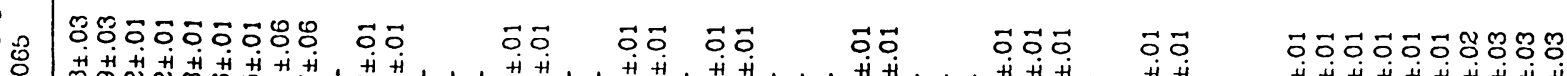

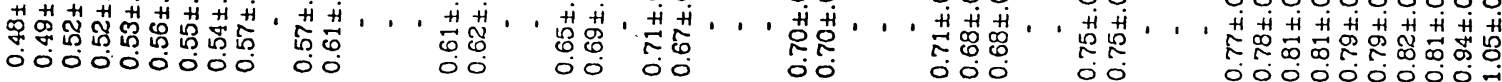

Tी

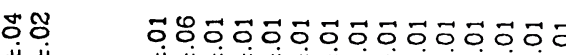

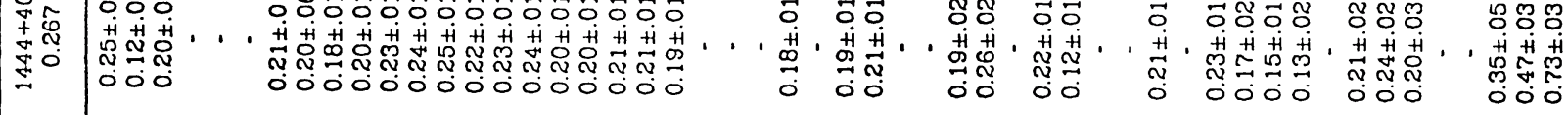

贵

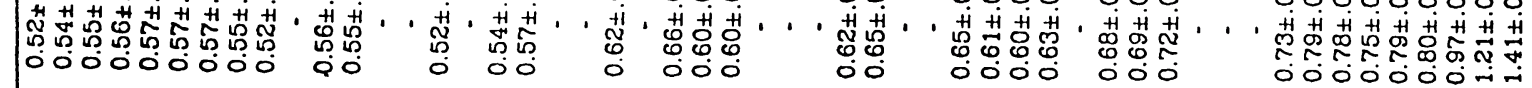

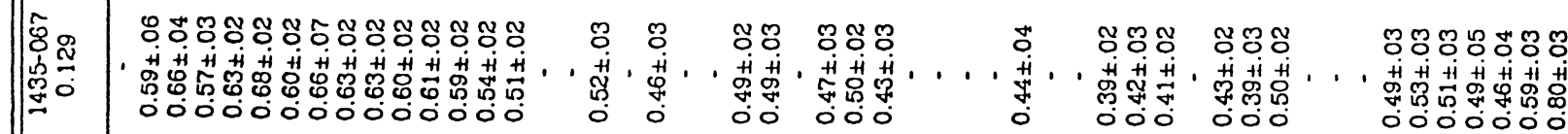

"

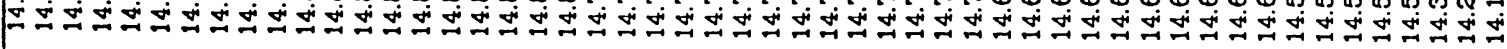

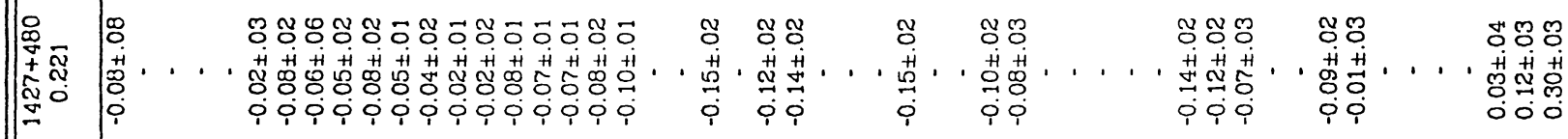

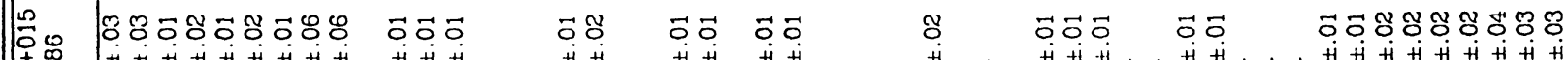

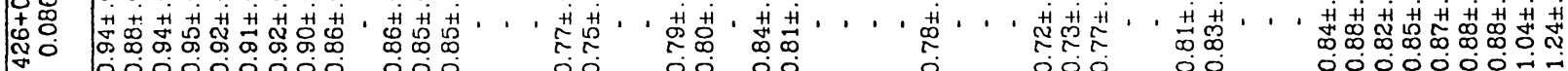

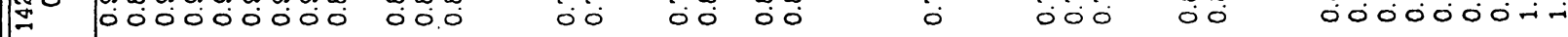

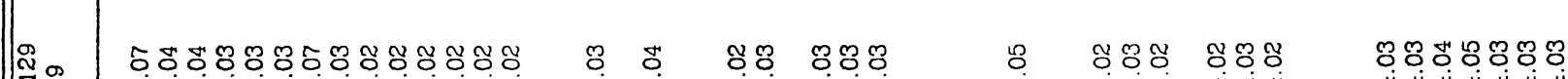

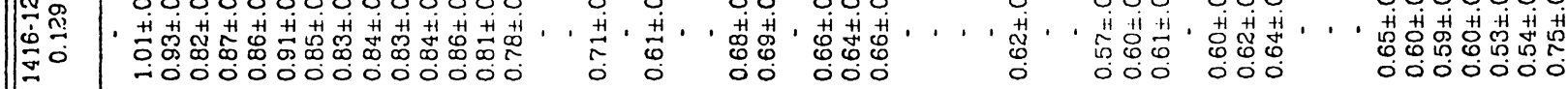

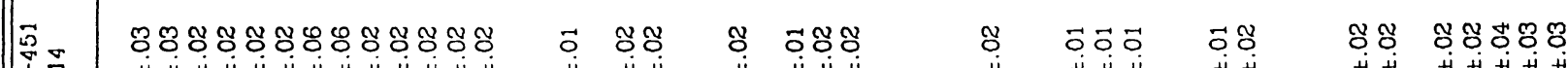

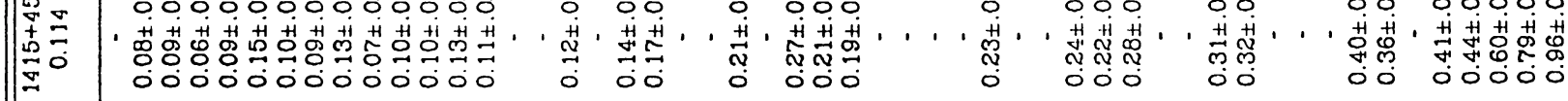

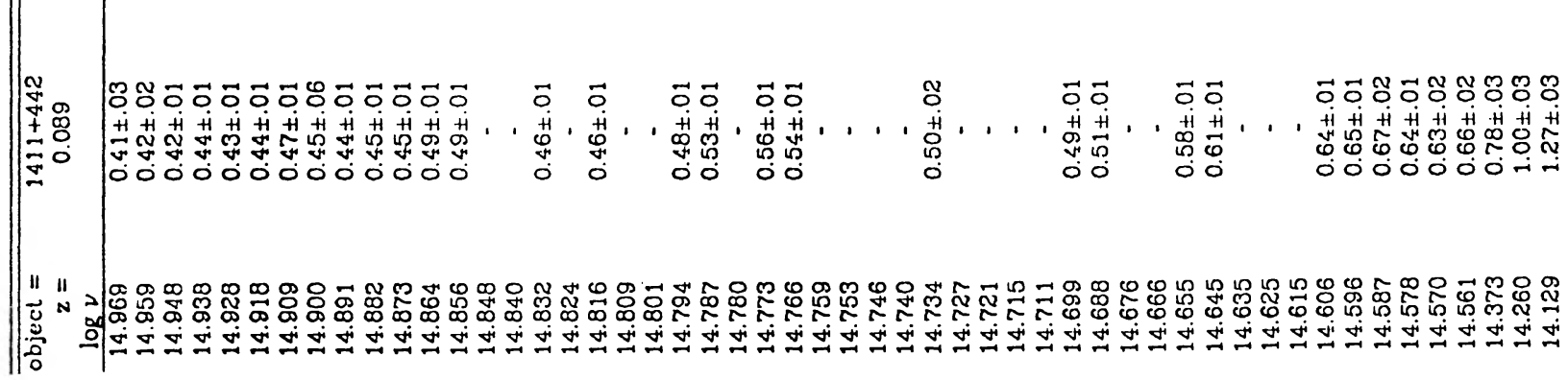




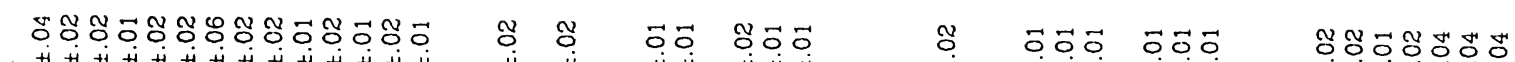

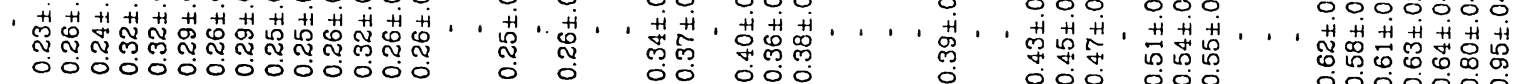

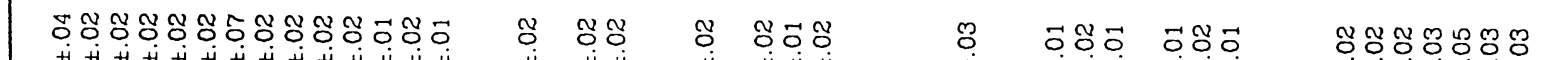

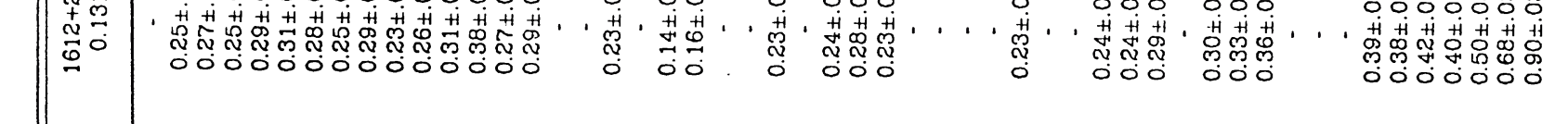

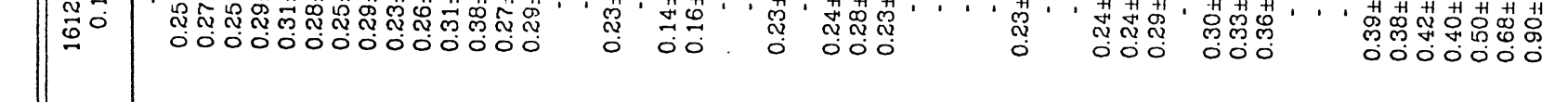

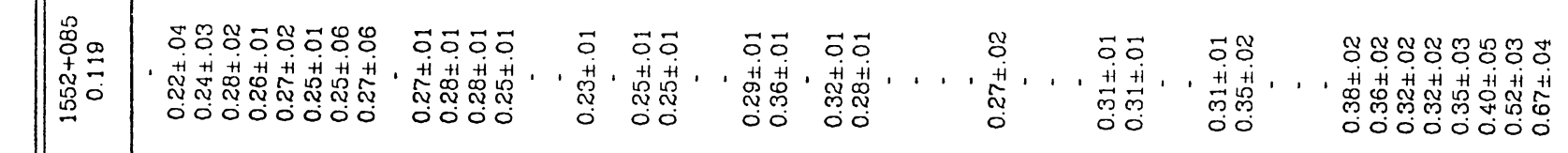

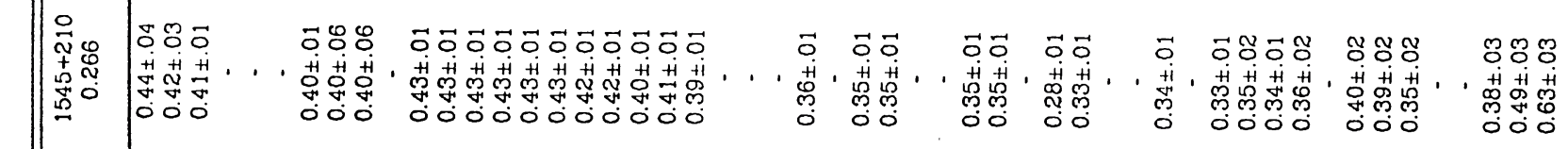

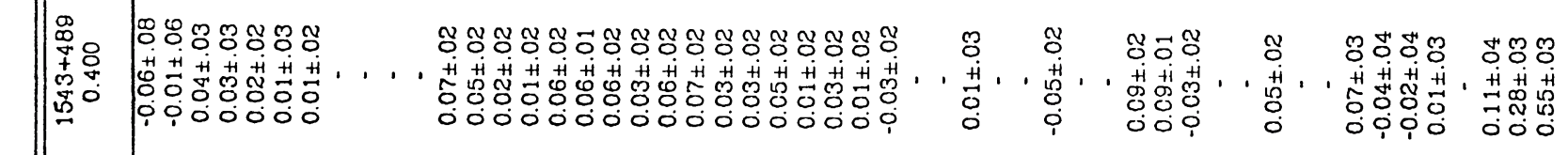

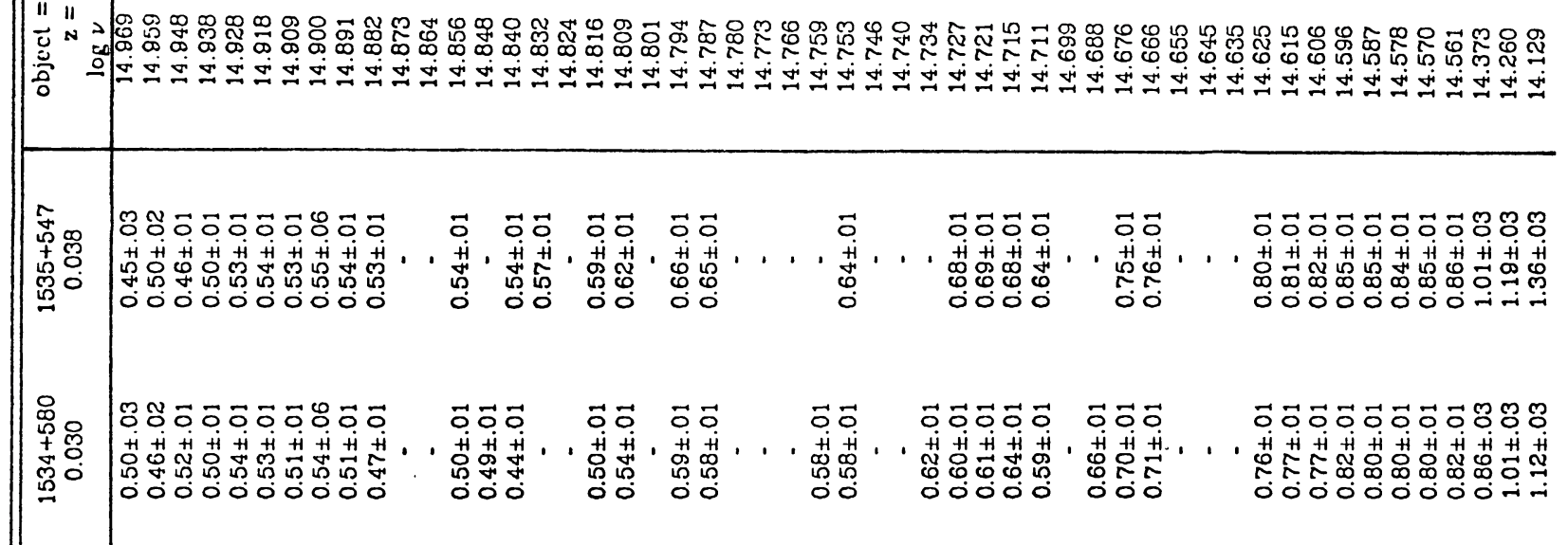

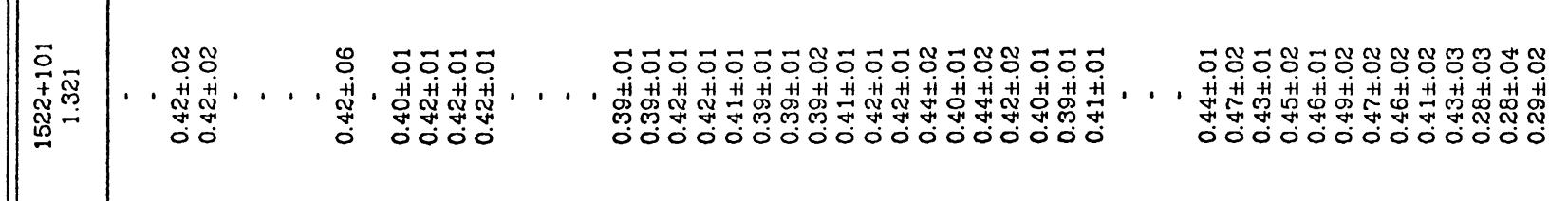
స్

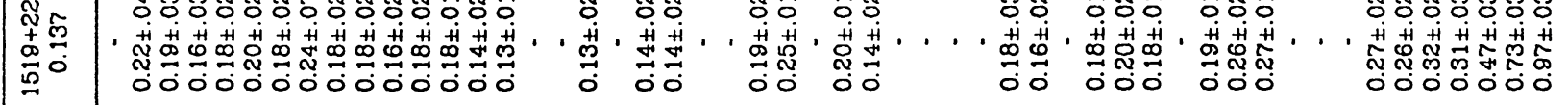

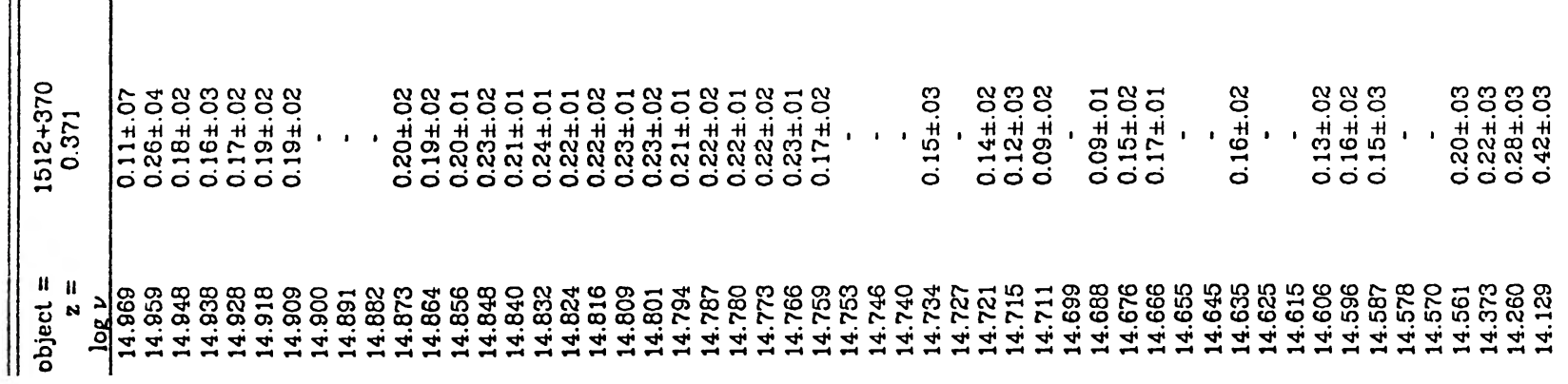




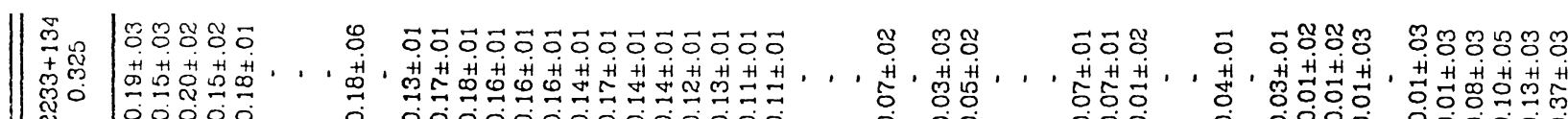

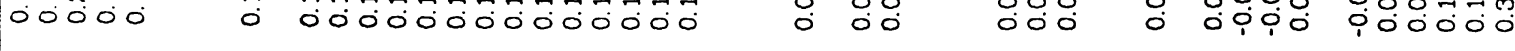

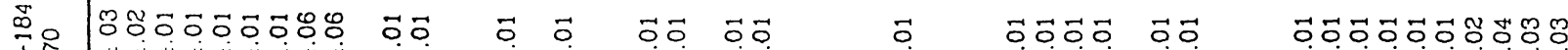

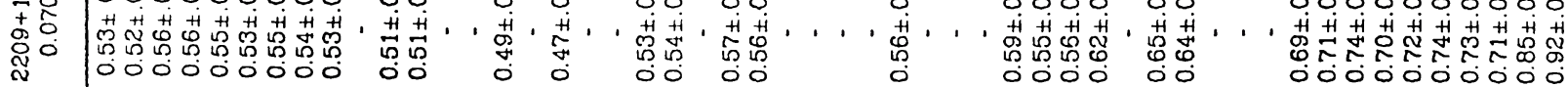

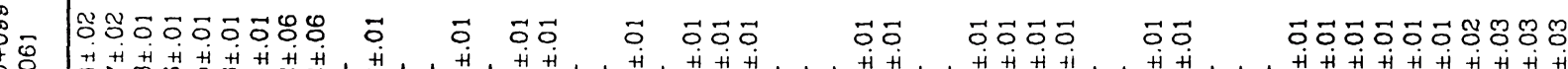

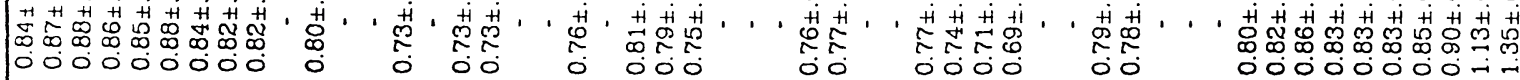

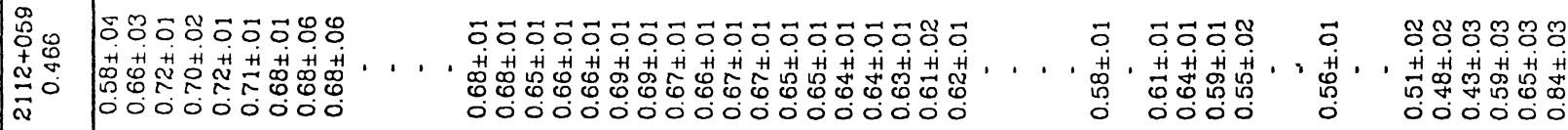

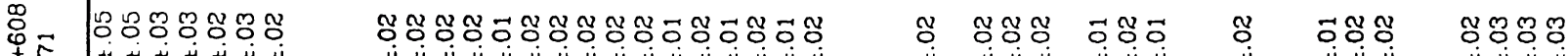

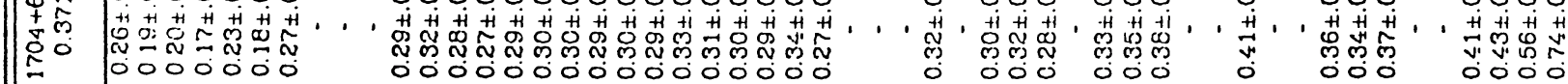

“ "

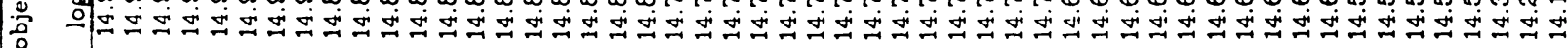

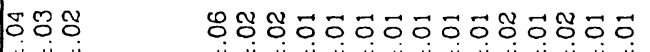

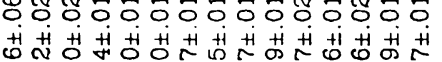

$\tilde{0} \quad \overrightarrow{0}$

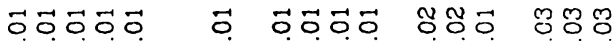

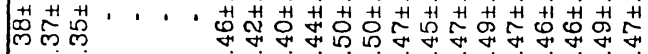

若.

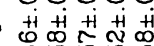

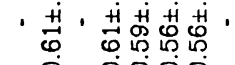

H

000

000000000000000

$\circ$ 0ं

00000

$\circ 0-$

\begin{tabular}{|c|c|c|c|c|}
\hline 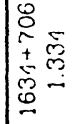 & 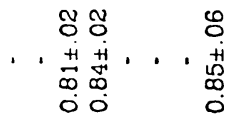 & 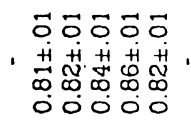 & 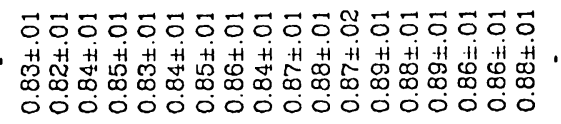 & 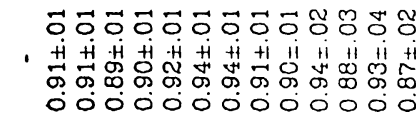 \\
\hline
\end{tabular}

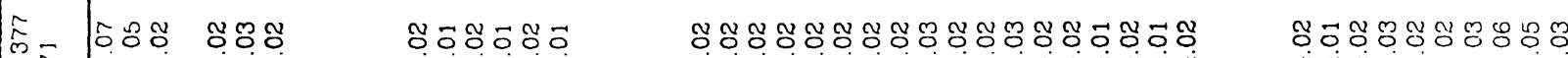

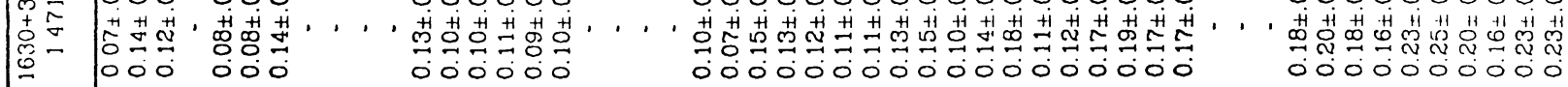

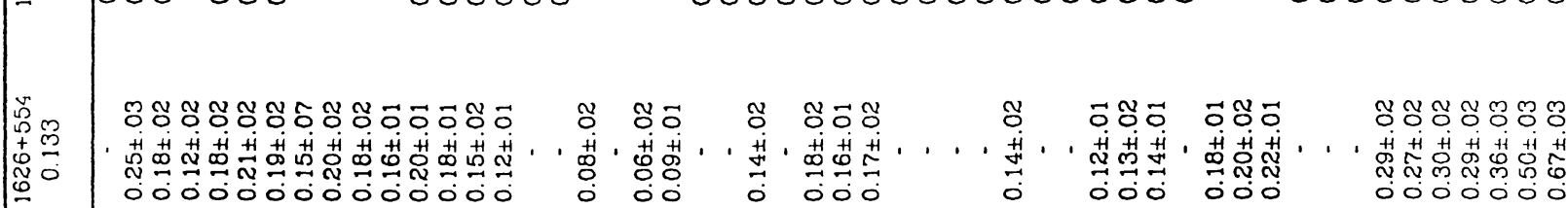

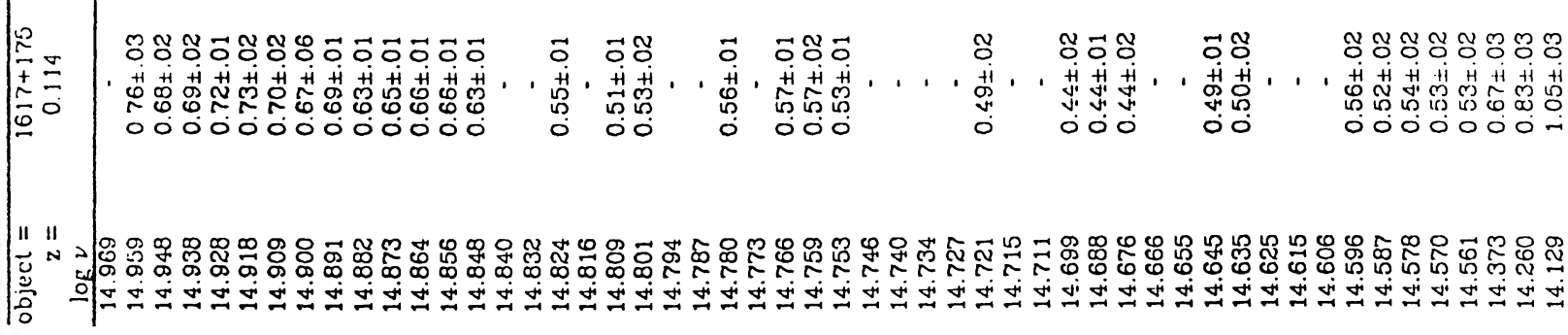




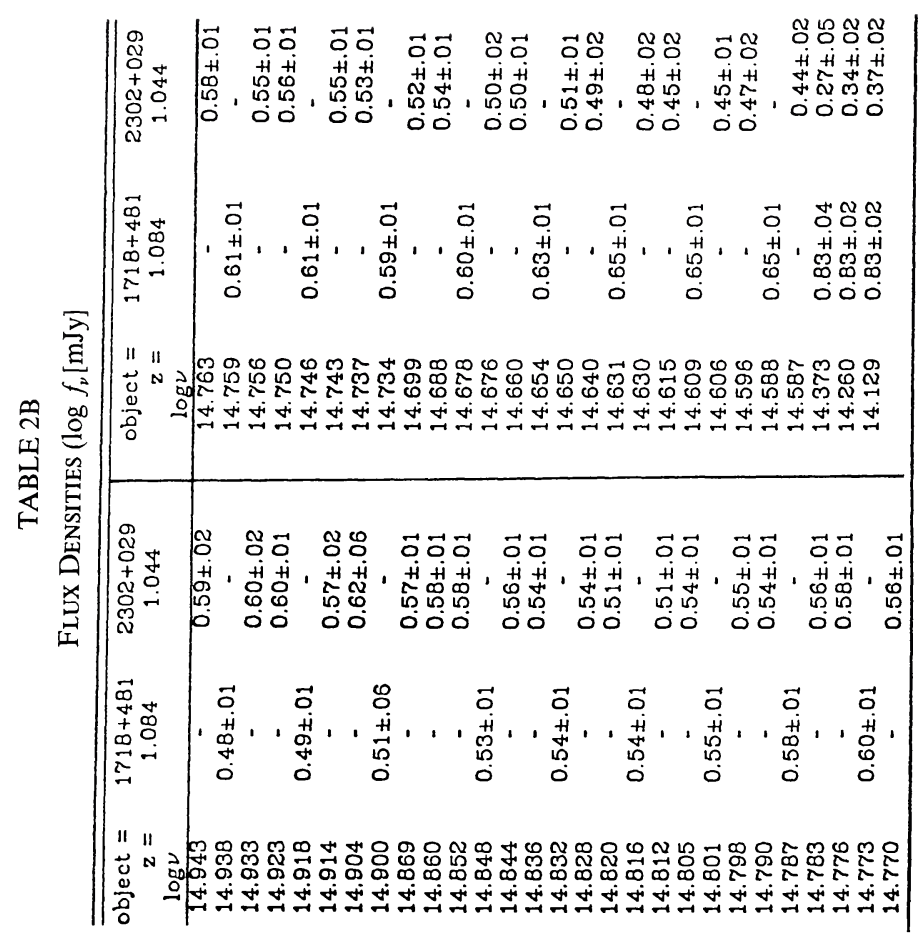

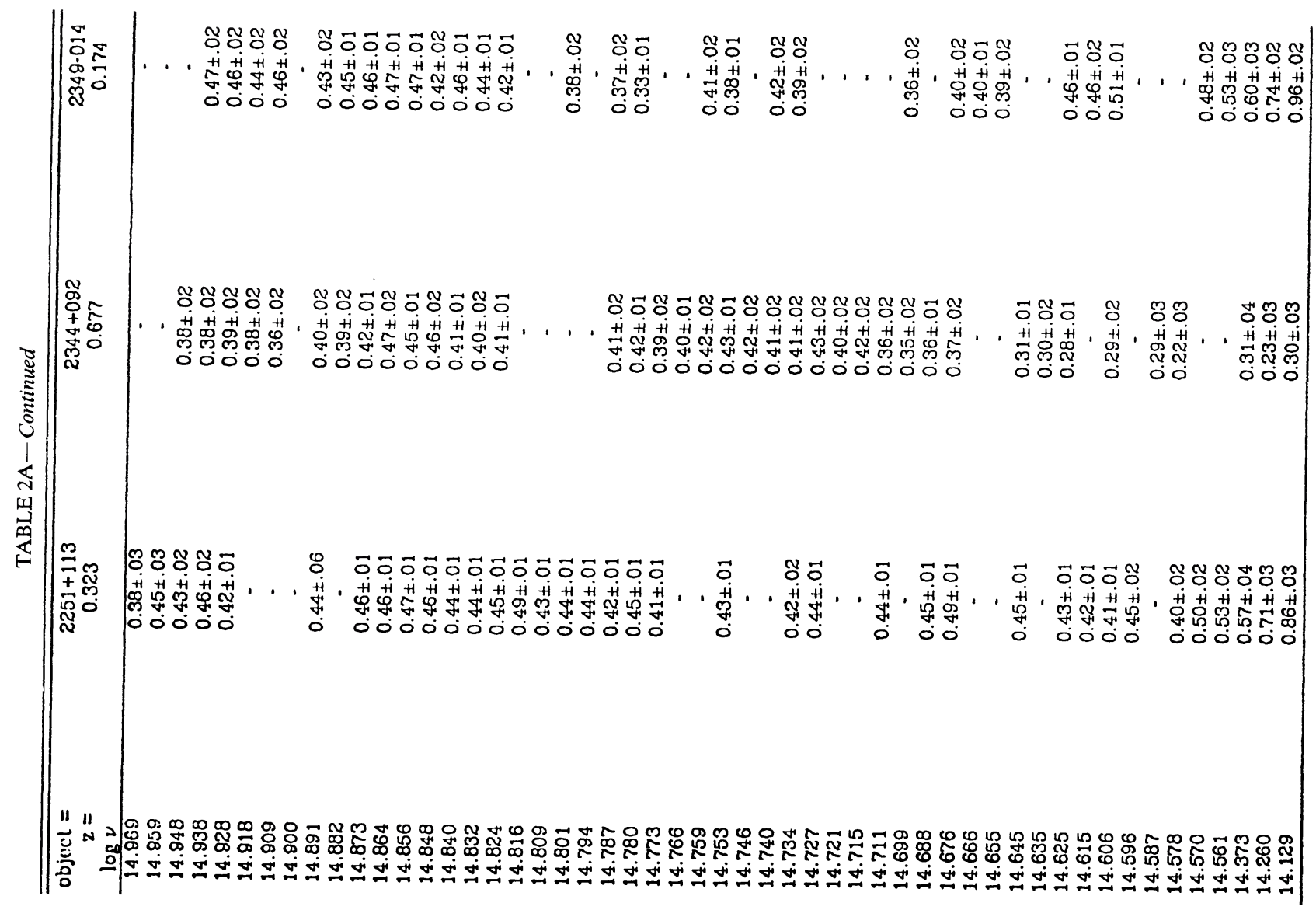


ments are included in Table 4, which also gives the accompanying $1.3-2.2 \mu \mathrm{m}$ observations. Only a few representative measurements of 3C 273 are given. This object has been observed at Palomar much more frequently than others as part of a regular monitoring program; extensive observations are given in NOBM, and more will be published as part of a study of the variability of quasars.

As indicated in Table 4, observations at 3.7 and $10.1 \mu \mathrm{m}$ were generally carried out at significantly different times than the observations made only at $1.3-2.2 \mu \mathrm{m}$ and with the multichannel spectrometer. In order to include these later measurements in the energy distributions of Figure 1, the observations at the longer wavelengths, which were always accompanied by repeated observations at 1.3-2.2 $\mu \mathrm{m}$, were adjusted so that the average slopes from 1.65 and $2.2 \mu \mathrm{m}$ to 3.7 or $10.1 \mu \mathrm{m}$ were those of the later epoch and the $2.2 \mu \mathrm{m}$ flux densities correspond to the earlier epoch of common infrared-visual observations. The maximum adjustments were of order $25 \%$. Since there may be real color variations in the quasars, this procedure potentially introduces an additional error in the plotted long-wavelength continua. The values tabulated in Table 4 are the observed flux densities.

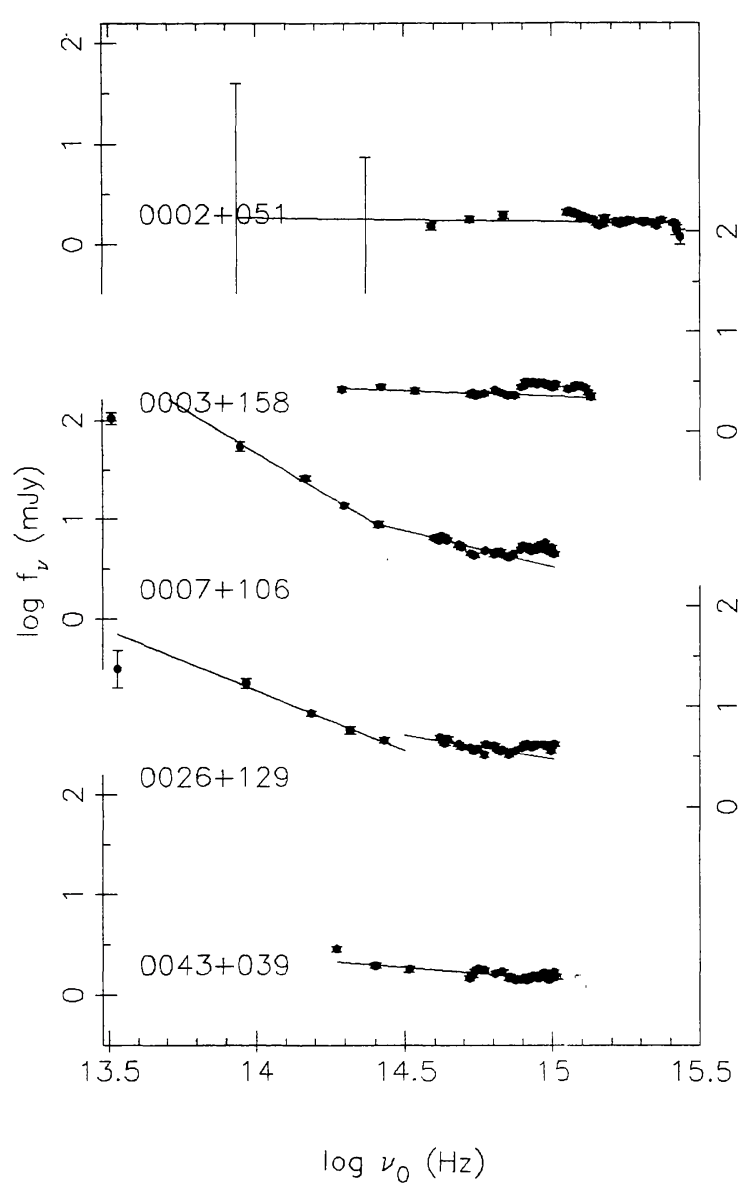

The bandwidths used in the near-infrared photometry are sufficiently broad that it was potentially necessary to correct the flux densities to account for the difference in continuum shape between the standard stars and the objects. If the continuum distributions of the standard stars follow a Rayleigh-Jeans law while the logarithm of the intrinsic continuum flux density of the objects decreases linearly with increasing frequency, the corrections for this effect range from $2 \%$ to $4 \%$. Mean corrections using these assumptions were applied to all the infrared observations.

TABLE 3

\begin{tabular}{r|r}
\multicolumn{2}{c}{ ContinuUm InTERVALS $(\mu \mathrm{m})$} \\
\hline \hline$<0.0970$ & $0.4000-0.4065$ \\
$0.0985-0.1015$ & $0.4135-0.4265$ \\
$0.1050-0.1175$ & $0.4410-0.4530$ \\
$0.1260-0.1375$ & $0.4605-0.4780$ \\
$0.1425-0.1500$ & $0.5050-0.5150$ \\
$0.1600-0.1625$ & $0.5365-0.5775$ \\
$0.1655-0.1845$ & $0.5975-0.6345$ \\
$0.1975-0.2700$ & $>0.6790$ \\
\hline
\end{tabular}

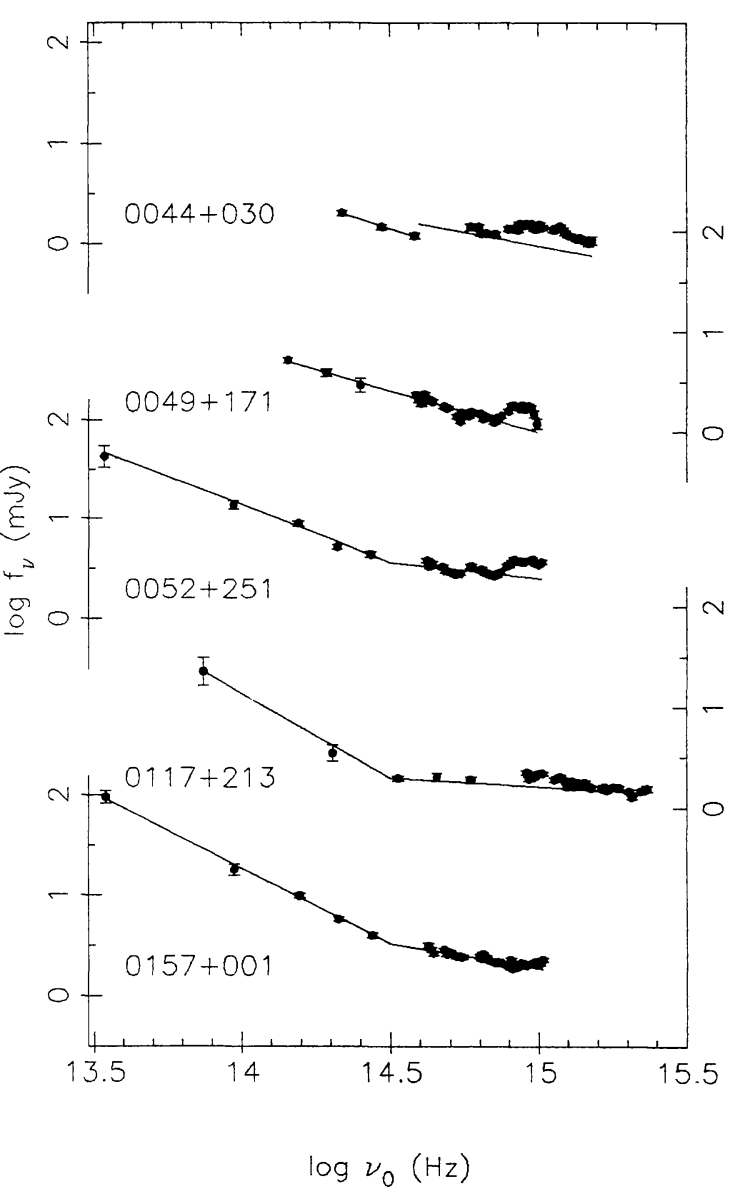

FIG. 1.-Plots of the continuum energy distributions for the 105 quasars measured in this program are given as a function of the rest frequency $\nu_{0}$. The ordinates for the top curve and the third and fifth curves from the top are given on the left. Those for the second and fourth curves from the top are given on the right. The upper limits shown are 3 standard deviations. 

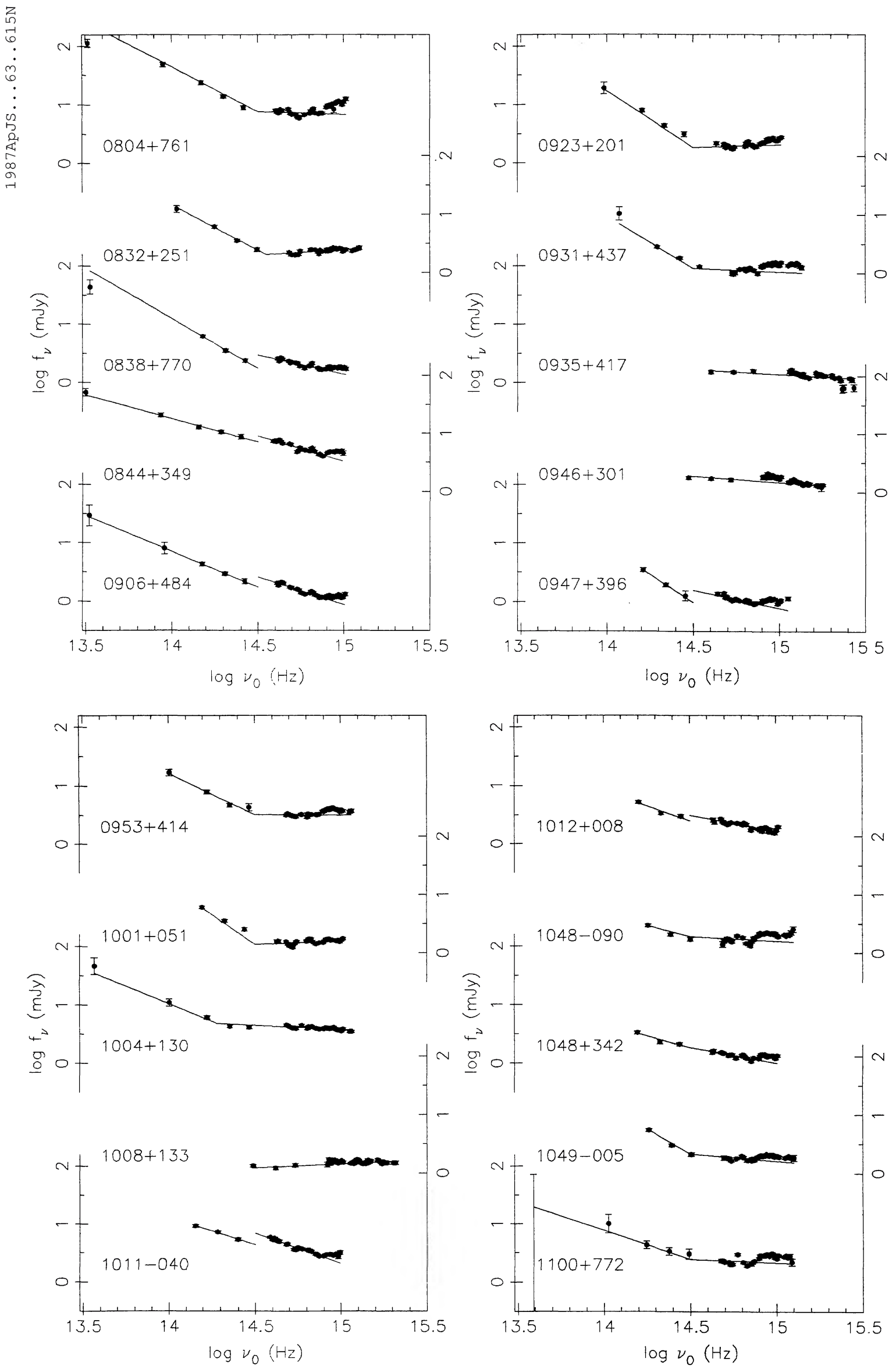

FIG. 1-Continued 

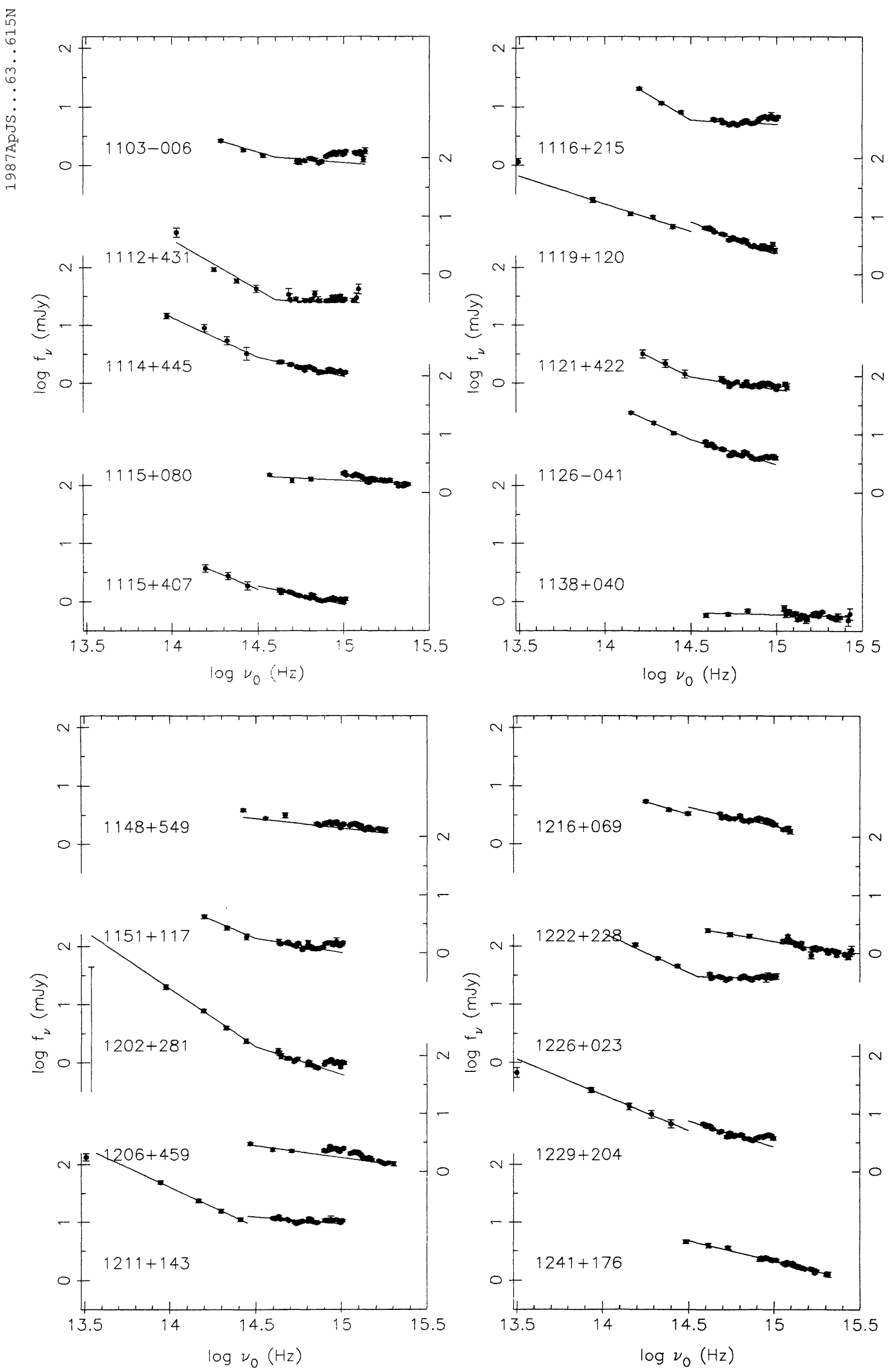

FIG. 1-Continued 

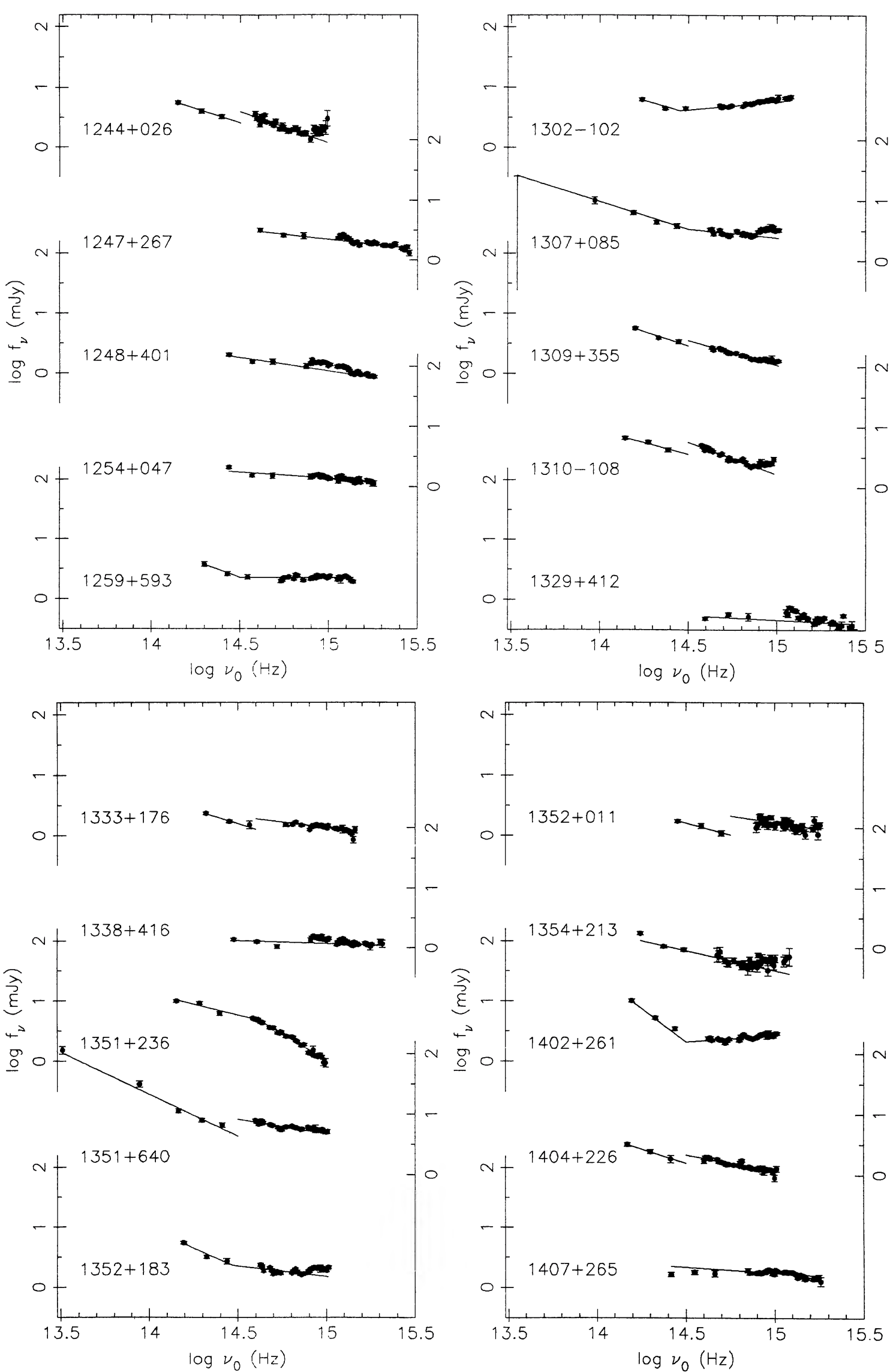

FIG. 1-Continued 

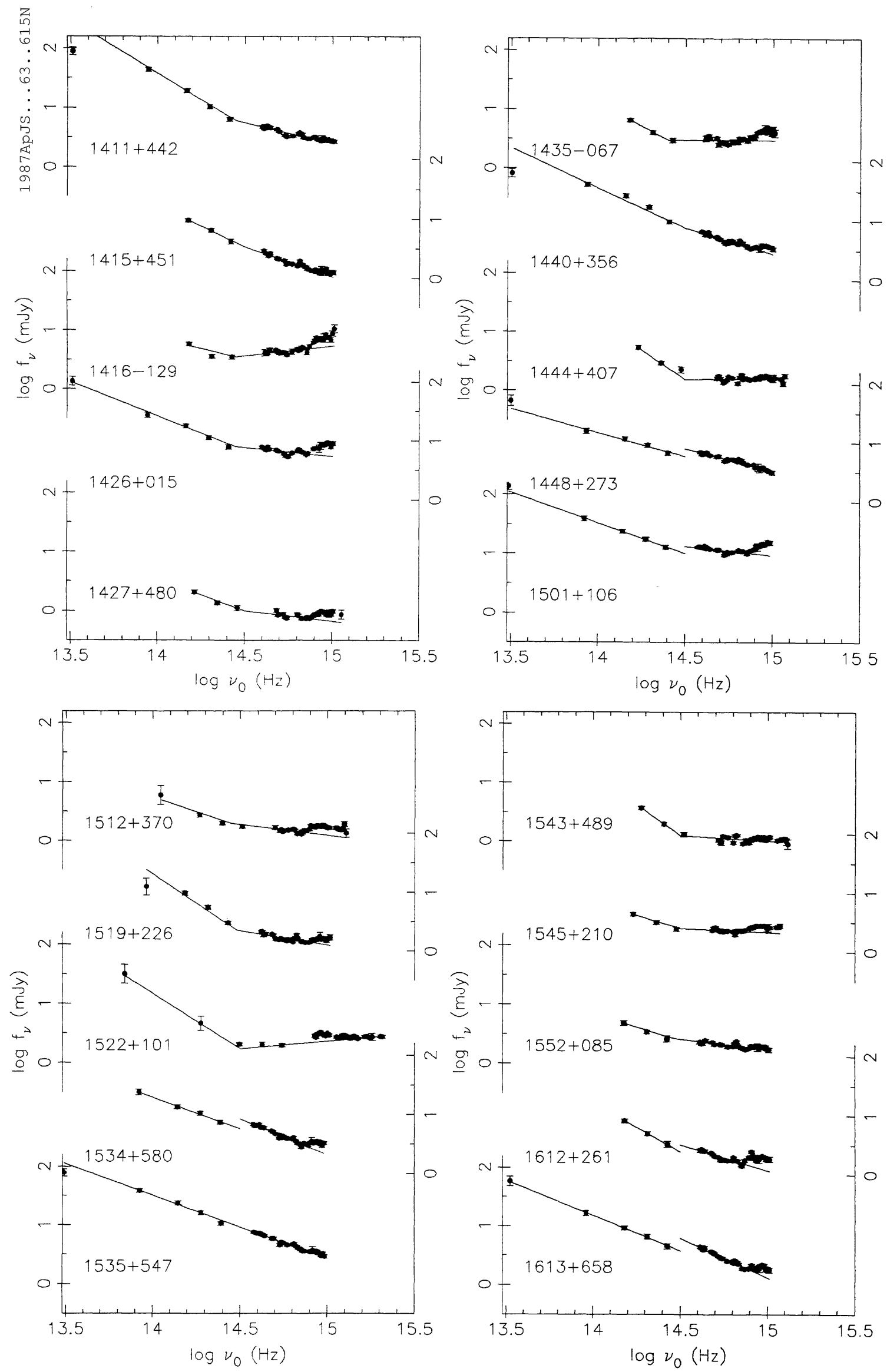

Fig. 1-Continued 

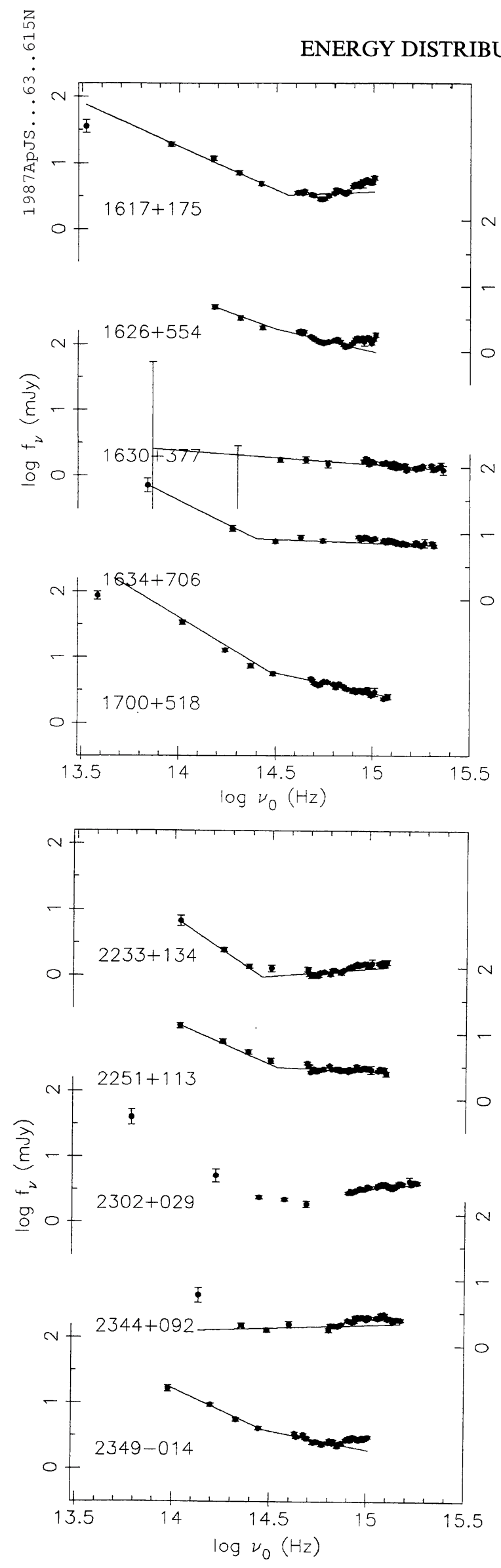

In the following, all frequencies will be the rest frequencies, $\nu_{0}$, unless specifically designated otherwise, and the flux densi. ties will be taken to be the adjusted values shown in Figure 1.

\section{DISCUSSION}

An examination of Figure 1 shows that a variety of continua is present, although the vast majority of the quasars have flux densities whose logarithms fall inversely with increasing frequency. Qualitatively, the observed continua of the majority of the quasars included in these observations can be divided into two categories:

1. The continua between 0.1 and $\sim 4.5 \mu \mathrm{m}$ of 78 of the quasars can be approximated by power-law continua plus a "3000 A bump" near $0.3 \mu \mathrm{m}$. An archetypal continuum of a quasar in this category is provided by PG $0157+001$. Of the 78 continua which can be adequately fitted by power laws, each of 58 can be fitted by two power-law slopes joining smoothly at a wavelength of about $1 \mu \mathrm{m}$, while each of the remaining continua can be fitted by a single power law.

2. For 26 quasars, the shorter wavelength observations obtained with the multichannel spectrometer were judged not to join smoothly onto those at longer wavelengths obtained with the infrared photometer. The quasar PG 1011-040 provides an example of one of these 26 quasars. As discussed below, we feel that the discontinuity which 
TABLE 4

LONG-WAVELENGTH INFRARED MEASUREMENTS

\begin{tabular}{|c|c|c|c|c|c|c|}
\hline \multirow[t]{2}{*}{ Object } & \multirow[t]{2}{*}{ Date } & \multicolumn{4}{|c|}{ Log flux density (mJy) } & \multirow[b]{2}{*}{$10.1 \mu \mathrm{m}$} \\
\hline & & $1.27 \mu \mathrm{m}$ & $1.65 \mu \mathrm{m}$ & $2.2 \mu \mathrm{m}$ & $3.7 \mu \mathrm{m}$ & \\
\hline $0002+051$ & $\begin{array}{c}3 J u n 82 \\
1 \text { Aug82 } \\
230 \text { ctB3 }\end{array}$ & $\begin{array}{l}0.23 \pm 0.04 \\
0.26 \pm 0.04 \\
0.21 \pm 0.03\end{array}$ & $\begin{array}{l}0.25 \pm 0.03 \\
0.23 \pm 0.03 \\
0.18 \pm 0.03\end{array}$ & $\begin{array}{l}0.24 \pm 0.04 \\
0.24 \pm 0.04 \\
0.24 \pm 0.03\end{array}$ & $\begin{array}{l}<.36 \\
<.79 \\
<.22\end{array}$ & $\begin{array}{l}<1.4 \\
<1.6\end{array}$ \\
\hline $0007+106$ & $\begin{array}{l}\text { 29Jul70 } \\
\text { 25Jul77 } \\
\text { 23JulB1 } \\
\text { 24JulB1 } \\
\text { 3NovB2 } \\
\text { 23JulB3 } \\
\text { 20AugB3 } \\
26 S e p 83 \\
\text { 21Nov83 } \\
\text { 15Sep84 } \\
\text { BOct84 }\end{array}$ & $\begin{array}{l}0.95 \pm 0.04 \\
0.78 \pm 0.03 \\
0.76 \pm 0.03 \\
0.79 \pm 0.04 \\
0.74 \pm 0.04 \\
0.75 \pm 0.04 \\
0.80 \pm 0.03 \\
0.73 \pm 0.04 \\
0.81 \pm 0.03\end{array}$ & $\begin{array}{l}0.89 \pm 0.05 \\
1.08 \pm 0.03 \\
0.97 \pm 0.03 \\
0.92 \pm 0.03 \\
0.93 \pm 0.03 \\
0.89 \pm 0.03 \\
0.89 \pm 0.03 \\
0.94 \pm 0.03 \\
0.89 \pm 0.03 \\
0.95 \pm 0.03\end{array}$ & $\begin{array}{l}1.14 \pm 0.04 \\
1.31 \pm 0.03 \\
1.28 \pm 0.03 \\
1.28 \pm 0.03 \\
1.23 \pm 0.03 \\
1.22 \pm 0.03 \\
1.19 \pm 0.03 \\
1.18 \pm 0.03 \\
1.22 \pm 0.03 \\
1.17 \pm 0.03 \\
1.23 \pm 0.03\end{array}$ & $\begin{array}{l}1.45 \pm 0.08 \\
1.54 \pm 0.07 \\
1.62 \pm 0.05 \\
1.56 \pm 0.04 \\
1.62 \pm 0.04 \\
1.46 \pm 0.04 \\
1.52 \pm 0.04 \\
1.51 \pm 0.04 \\
1.45 \pm 0.05 \\
1.50 \pm 0.04\end{array}$ & $\begin{array}{l}2.02 \pm 0.12 \\
1.90 \pm 0.06 \\
\\
1.88 \pm 0.08 \\
1.82 \pm 0.09 \\
1.87 \pm 0.07\end{array}$ \\
\hline $0026+129$ & $\begin{array}{l}170 c t 75 \\
\text { 22Jul81 }\end{array}$ & $0.65 \pm 0.03$ & $\begin{array}{l}0.84 \pm 0.03 \\
0.69 \pm 0.04\end{array}$ & $\begin{array}{l}0.98 \pm 0.02 \\
0.88 \pm 0.03\end{array}$ & $\begin{array}{l}1.28 \pm 0.08 \\
1.23 \pm 0.05\end{array}$ & $\begin{array}{l}1.55 \pm 0.12 \\
1.37 \pm 0.19\end{array}$ \\
\hline $0052+251$ & 21Jul81 & $0.57 \pm 0.03$ & $0.65 \pm 0.04$ & $0.89 \pm 0.03$ & $1.09 \pm 0.04$ & $1.59 \pm 0.11$ \\
\hline $0117+213$ & $230 \operatorname{ct} 83$ & $0.34 \pm 0.03$ & $0.37 \pm 0.03$ & $0.30 \pm 0.03$ & $0.48 \pm 0.08$ & $1.29 \pm 0.12$ \\
\hline $0157+001$ & BNov81 & $0.57 \pm 0.03$ & $0.73 \pm 0.03$ & $0.97 \pm 0.03$ & $1.24 \pm 0.06$ & $1.96 \pm 0.06$ \\
\hline $0804+761$ & BNov81 & $0.95 \pm 0.03$ & $1.11 \pm 0.03$ & $1.40 \pm 0.03$ & $1.71 \pm 0.04$ & $2.07 \pm 0.07$ \\
\hline $0832+251$ & 21Nov83 & $0.34 \pm 0.03$ & $0.49 \pm 0.03$ & $0.76 \pm 0.03$ & $1.07 \pm 0.06$ & \\
\hline $0838+770$ & 21Nov85 & $0.36 \pm 0.03$ & $0.49 \pm 0.03$ & $0.68 \pm 0.03$ & $0.80 \pm 0.06$ & $1.61 \pm 0.10$ \\
\hline $0844+349$ & $\begin{array}{l}180 \operatorname{ct} 80 \\
8 \text { Nov81 }\end{array}$ & $0.90 \pm 0.03$ & $0.98 \pm 0.03$ & $\begin{array}{l}1.12 \pm 0.04 \\
1.10 \pm 0.03\end{array}$ & $\begin{array}{l}1.39 \pm 0.05 \\
1.28 \pm 0.04\end{array}$ & $1.69 \pm 0.06$ \\
\hline $0906+484$ & 14 May76 & $0.37 \pm 0.05$ & $0.50 \pm 0.04$ & $0.76 \pm 0.04$ & $1.00 \pm 0.08$ & $1.55 \pm 0.16$ \\
\hline $0953+414$ & 8 Nov81 & $0.53 \pm 0.03$ & $0.63 \pm 0.03$ & $0.89 \pm 0.03$ & $1.19 \pm 0.06$ & \\
\hline $1004+130$ & $\begin{array}{c}\text { BFeb77 } \\
21 \text { Nov83 }\end{array}$ & $0.62 \pm 0.03$ & $\begin{array}{l}0.61 \pm 0.04 \\
0.65 \pm 0.03\end{array}$ & $\begin{array}{l}0.74 \pm 0.04 \\
0.79 \pm 0.03\end{array}$ & $0.96 \pm 0.04$ & $1.63 \pm 0.12$ \\
\hline $1100+772$ & 28 May77 & $0.41 \pm 0.04$ & $0.44 \pm 0.03$ & $0.64 \pm 0.02$ & $0.98 \pm 0.12$ & $<1.8$ \\
\hline $1112+431$ & 21Nov83 & $-0.14 \pm 0.03$ & $0.02 \pm 0.03$ & $0.23 \pm 0.03$ & $0.54 \pm 0.06$ & \\
\hline $1114+445$ & 21 Nov83 & $0.55 \pm 0.03$ & $0.66 \pm 0.03$ & $0.91 \pm 0.03$ & $1.17 \pm 0.04$ & \\
\hline $1119+120$ & 11 May81 & $0.88 \pm 0.03$ & $0.99 \pm 0.03$ & $1.10 \pm 0.03$ & $1.33 \pm 0.04$ & $1.97 \pm 0.06$ \\
\hline $1202+281$ & $\begin{array}{l}\text { 12May81 } \\
\text { 23JulB1 }\end{array}$ & $\begin{array}{l}0.33 \pm 0.03 \\
0.33 \pm 0.05\end{array}$ & $\begin{array}{l}0.49 \pm 0.03 \\
0.47 \pm 0.03\end{array}$ & $\begin{array}{l}0.81 \pm 0.03 \\
0.81 \pm 0.03\end{array}$ & $1.22 \pm 0.04$ & $<1.5$ \\
\hline $1211+143$ & 13May81 & $1.02 \pm 0.03$ & $1.17 \pm 0.03$ & $1.42 \pm 0.03$ & $1.69 \pm 0.03$ & $2.12 \pm 0.06$ \\
\hline $1226+023$ & $\begin{array}{c}\text { 13May76 } \\
\text { 29May77 } \\
\text { 03Jul79 } \\
11 \text { May81 } \\
22 J u 181 \\
25 J u 183 \\
5 J u n 85\end{array}$ & $\begin{array}{l}1.51 \pm 0.04 \\
1.52 \pm 0.03 \\
1.53 \pm 0.04 \\
1.59 \pm 0.03 \\
1.57 \pm 0.03 \\
1.63 \pm 0.04 \\
1.51 \pm 0.03\end{array}$ & $\begin{array}{l}1.64 \pm 0.03 \\
1.64 \pm 0.03 \\
1.69 \pm 0.03 \\
1.70 \pm 0.03 \\
1.70 \pm 0.04 \\
1.76 \pm 0.05 \\
1.63 \pm 0.03\end{array}$ & $\begin{array}{l}1.91 \pm 0.03 \\
1.90 \pm 0.03 \\
1.92 \pm 0.03 \\
1.95 \pm 0.03 \\
1.95 \pm 0.04 \\
1.99 \pm 0.04 \\
1.91 \pm 0.03\end{array}$ & $\begin{array}{l}2.18 \pm 0.04 \\
2.06 \pm 0.03 \\
2.00 \pm 0.06 \\
2.18 \pm 0.03 \\
2.26 \pm 0.05 \\
2.17 \pm 0.03\end{array}$ & $\begin{array}{l}2.52 \pm 0.07 \\
2.55 \pm 0.06 \\
2.52 \pm 0.05 \\
2.50 \pm 0.06 \\
2.48 \pm 0.06 \\
2.48 \pm 0.05\end{array}$ \\
\hline $1229+204$ & 11 May81 & $0.78 \pm 0.03$ & $0.93 \pm 0.03$ & $1.12 \pm 0.03$ & $1.38 \pm 0.04$ & $1.67 \pm 0.08$ \\
\hline $1307+085$ & $\begin{array}{l}\text { 21Jul81 } \\
\text { 22Jul81 }\end{array}$ & $\begin{array}{l}0.55 \pm 0.03 \\
0.55 \pm 0.03\end{array}$ & $\begin{array}{l}0.63 \pm 0.04 \\
0.64 \pm 0.04\end{array}$ & $\begin{array}{l}0.83 \pm 0.04 \\
0.85 \pm 0.03\end{array}$ & $1.01 \pm 0.06$ & $<1.4$ \\
\hline $1351+640$ & $\begin{array}{l}\text { 14May76 } \\
\text { 21JulB1 }\end{array}$ & $\begin{array}{l}0.81 \pm 0.04 \\
0.83 \pm 0.03\end{array}$ & $\begin{array}{l}0.84 \pm 0.03 \\
0.87 \pm 0.04\end{array}$ & $\begin{array}{l}1.03 \pm 0.03 \\
1.05 \pm 0.03\end{array}$ & $\begin{array}{l}1.31 \pm 0.05 \\
1.50 \pm 0.05\end{array}$ & $2.06 \pm 0.06$ \\
\hline $1411+442$ & 13May81 & $0.75 \pm 0.03$ & $0.92 \pm 0.03$ & $1.24 \pm 0.03$ & $1.59 \pm 0.03$ & $1.89 \pm 0.06$ \\
\hline $1426+015$ & 24JulB1 & $0.77 \pm 0.03$ & $0.94 \pm 0.04$ & $1.20 \pm 0.03$ & $1.37 \pm 0.04$ & $1.94 \pm 0.08$ \\
\hline $1440+356$ & $\begin{array}{c}\text { 12May81 } \\
\text { 13MayB1 } \\
\text { 9MayB4 }\end{array}$ & $\begin{array}{l}0.99 \pm 0.03 \\
0.98 \pm 0.03 \\
1.00 \pm 0.03\end{array}$ & $\begin{array}{l}1.19 \pm 0.03 \\
1.19 \pm 0.03 \\
1.15 \pm 0.03\end{array}$ & $\begin{array}{l}1.41 \pm 0.03 \\
1.41 \pm 0.03 \\
1.38 \pm 0.03\end{array}$ & $\begin{array}{l}1.60 \pm 0.03 \\
1.58 \pm 0.04\end{array}$ & $1.77 \pm 0.07$ \\
\hline $1448+273$ & $\begin{array}{l}\text { 12May81 } \\
\text { 13MayB1 }\end{array}$ & $\begin{array}{l}0.80 \pm 0.03 \\
0.78 \pm 0.03\end{array}$ & $\begin{array}{l}0.93 \pm 0.03 \\
0.92 \pm 0.03\end{array}$ & $\begin{array}{l}1.07 \pm 0.03 \\
1.08 \pm 0.03\end{array}$ & $1.18 \pm 0.04$ & $1.68 \pm 0.09$ \\
\hline $1501+106$ & $\begin{array}{l}\text { 12May81 } \\
\text { 22JulB1 }\end{array}$ & $\begin{array}{l}1.07 \pm 0.03 \\
1.07 \pm 0.03\end{array}$ & $\begin{array}{l}1.23 \pm 0.03 \\
1.24 \pm 0.04\end{array}$ & $\begin{array}{l}1.41 \pm 0.03 \\
1.43 \pm 0.03\end{array}$ & $\begin{array}{l}1.61 \pm 0.03 \\
1.62 \pm 0.04\end{array}$ & $2.16 \pm 0.07$ \\
\hline $1512+370$ & 30 May77 & & & $0.38 \pm 0.05$ & $0.72 \pm 0.14$ & \\
\hline
\end{tabular}


TABLE 4-Continued

\begin{tabular}{|c|c|c|c|c|c|c|}
\hline \multirow[t]{2}{*}{ Object } & \multirow[t]{2}{*}{ Date } & \multicolumn{4}{|c|}{ Log flux density (mJy) } & \multirow[b]{2}{*}{$10.1 \mu \mathrm{m}$} \\
\hline & & $1.27 \mu \mathrm{m}$ & $1.65 \mu \mathrm{m}$ & $2.2 \mu \mathrm{m}$ & $3.7 \mu \mathrm{m}$ & \\
\hline $1519+226$ & $16 \mathrm{Jun} 78$ & $0.49 \pm 0.04$ & $0.73 \pm 0.04$ & $0.97 \pm 0.03$ & $1.06 \pm 0.12$ & \\
\hline $1522+101$ & 1JunB2 & $0.24 \pm 0.04$ & $0.38 \pm 0.03$ & $0.32 \pm 0.04$ & $0.60 \pm 0.10$ & $1.43 \pm 0.12$ \\
\hline $1534+580$ & 22Apr83 & $0.86 \pm 0.03$ & $0.98 \pm 0.03$ & $1.15 \pm 0.03$ & $1.34 \pm 0.03$ & \\
\hline $1535+547$ & $\begin{array}{l}\text { 12Apr71 } \\
12 \text { May81 } \\
13 \text { May81 }\end{array}$ & $\begin{array}{l}1.01 \pm 0.03 \\
1.01 \pm 0.03\end{array}$ & $\begin{array}{l}1.05 \pm 0.04 \\
1.20 \pm 0.03 \\
1.41 \pm 0.03\end{array}$ & $\begin{array}{l}1.27 \pm 0.03 \\
1.39 \pm 0.03 \\
1.90 \pm 0.06\end{array}$ & $\begin{array}{l}1.46 \pm 0.06 \\
1.59 \pm 0.03\end{array}$ & \\
\hline $1612+261$ & $\begin{array}{l}\text { 14May76 } \\
15 \text { May76 }\end{array}$ & $0.55 \pm 0.04$ & $0.71 \pm 0.04$ & $\begin{array}{l}0.93 \pm 0.03 \\
0.90 \pm 0.03\end{array}$ & $1.12 \pm 0.10$ & $1.59 \pm 0.11$ \\
\hline $1613 \pm 658$ & 21Jul81 & $0.66 \pm 0.03$ & $0.80 \pm 0.04$ & $1.03 \pm 0.03$ & $1.24 \pm 0.04$ & $1.78 \pm 0.08$ \\
\hline $1617+175$ & 18Aug81 & $0.63 \pm 0.03$ & $0.81 \pm 0.03$ & $1.09 \pm 0.03$ & $1.29 \pm 0.04$ & $1.56 \pm 0.10$ \\
\hline $1630+377$ & 18Aug81 & $0.26 \pm 0.04$ & $0.32 \pm 0.03$ & $0.20 \pm 0.03$ & $<.45$ & $<1.7$ \\
\hline $1634+706$ & $\begin{array}{l}\text { 12May81 } \\
\text { 21JulB1 }\end{array}$ & $\begin{array}{l}0.81 \pm 0.03 \\
0.83 \pm 0.03\end{array}$ & $\begin{array}{l}0.97 \pm 0.03 \\
0.98 \pm 0.04\end{array}$ & $\begin{array}{l}0.83 \pm 0.03 \\
0.03: 0.04\end{array}$ & $1.03 \pm 0.04$ & $1.69 \pm 0.11$ \\
\hline $1700+518$ & $\begin{array}{l}\text { 12May81 } \\
\text { 13May81 }\end{array}$ & $\begin{array}{l}0.65 \pm 0.03 \\
0.63 \pm 0.03\end{array}$ & $\begin{array}{l}0.82 \pm 0.03 \\
0.81 \pm 0.03\end{array}$ & $\begin{array}{l}1.10 \pm 0.03 \\
1.11 \pm 0.03\end{array}$ & $1.49 \pm 0.03$ & $1.88 \pm 0.06$ \\
\hline $1704+608$ & $\begin{array}{l}140 c t 76 \\
24 J u l 81\end{array}$ & $\begin{array}{l}0.50 \pm 0.04 \\
0.45 \pm 0.04\end{array}$ & $\begin{array}{l}0.59 \pm 0.03 \\
0.52 \pm 0.04\end{array}$ & $\begin{array}{l}0.79 \pm 0.03 \\
0.74 \pm 0.04\end{array}$ & $1.16 \pm 0.06$ & $\begin{array}{l}1.67 \pm 0.08 \\
1.60 \pm 0.10\end{array}$ \\
\hline $1718+481$ & $\begin{array}{l}\text { 12May81 } \\
\text { 22Jul81 }\end{array}$ & $\begin{array}{l}0.82 \pm 0.03 \\
0.80 \pm 0.03\end{array}$ & $\begin{array}{l}0.81 \pm 0.03 \\
0.81 \pm 0.04\end{array}$ & $\begin{array}{l}0.80 \pm 0.03 \\
0.82 \pm 0.03\end{array}$ & $0.98 \pm 0.04$ & $1.43 \pm 0.12$ \\
\hline $2112+059$ & $\begin{array}{l}\text { 23Jul81 } \\
\text { 17Aug81 }\end{array}$ & $\begin{array}{l}0.48 \pm 0.04 \\
0.45 \pm 0.03\end{array}$ & $\begin{array}{l}0.58 \pm 0.03 \\
0.59 \pm 0.03\end{array}$ & $\begin{array}{l}0.82 \pm 0.03 \\
0.82 \pm 0.03\end{array}$ & $\begin{array}{l}1.18 \pm 0.05 \\
1.27 \pm 0.07\end{array}$ & $1.52 \pm 0.13$ \\
\hline $2130+099$ & $\begin{array}{l}\text { 26Jul70 } \\
\text { 22Jul81 } \\
\text { 22Apr83 }\end{array}$ & $\begin{array}{l}0.91 \pm 0.03 \\
0.90 \pm 0.03\end{array}$ & $\begin{array}{l}1.31 \pm 0.03 \\
1.14 \pm 0.04 \\
1.11 \pm 0.03\end{array}$ & $\begin{array}{l}1.53 \pm 0.02 \\
1.40 \pm 0.03 \\
1.38 \pm 0.03\end{array}$ & $\begin{array}{l}1.67 \pm 0.08 \\
1.64 \pm 0.03 \\
1.68 \pm 0.03\end{array}$ & $2.19 \pm 0.06$ \\
\hline $2209+184$ & 17AugB1 & $0.60 \pm 0.03$ & $0.75 \pm 0.03$ & $0.85 \pm 0.03$ & $0.97 \pm 0.05$ & $1.26 \pm 0.14$ \\
\hline $2233+134$ & $\begin{array}{l}\text { 14Sep81 } \\
\text { 3Jun82 }\end{array}$ & $\begin{array}{l}-0.01 \pm 0.05 \\
-0.01 \pm 0.05\end{array}$ & $\begin{array}{l}0.07 \pm 0.03 \\
0.09 \pm 0.03\end{array}$ & $\begin{array}{l}0.31 \pm 0.02 \\
0.36 \pm 0.03\end{array}$ & $\begin{array}{l}0.58 \pm 0.10 \\
0.80 \pm 0.08\end{array}$ & \\
\hline $2251+113$ & $\begin{array}{l}30 J u n 69 \\
16 J u n 78 \\
14 \operatorname{Sep} 81\end{array}$ & $\begin{array}{l}0.53 \pm 0.04 \\
0.53 \pm 0.03\end{array}$ & $\begin{array}{l}0.67 \pm 0.04 \\
0.67 \pm 0.02\end{array}$ & $\begin{array}{l}0.85 \pm 0.08 \\
0.85 \pm 0.04 \\
0.89 \pm 0.02\end{array}$ & $\begin{array}{c}<1.5 \\
0.94 \pm 0.12 \\
1.12 \pm 0.04\end{array}$ & \\
\hline $2302+029$ & $\begin{array}{c}\text { 24Jul81 } \\
\text { 14Sep81 } \\
\text { 1Jun82 }\end{array}$ & $\begin{array}{l}0.30 \pm 0.04 \\
0.30 \pm 0.03 \\
0.33 \pm 0.04\end{array}$ & $\begin{array}{l}0.27 \pm 0.04 \\
0.29 \pm 0.03 \\
0.26 \pm 0.03\end{array}$ & $\begin{array}{l}0.34 \pm 0.04 \\
0.34 \pm 0.03 \\
0.36 \pm 0.04\end{array}$ & $\begin{array}{l}0.64 \pm 0.10 \\
0.40 \pm 0.16 \\
0.66 \pm 0.69\end{array}$ & $\begin{array}{l}1.33 \pm 0.20 \\
1.56 \pm 0.10\end{array}$ \\
\hline $2344+092$ & $14 \operatorname{Sep} 81$ & $0.20 \pm 0.03$ & $0.19 \pm 0.03$ & $0.28 \pm 0.03$ & $0.75 \pm 0.11$ & \\
\hline $2349-014$ & $14 \operatorname{Sep} 81$ & $0.62 \pm 0.03$ & $0.76 \pm 0.02$ & $0.98 \pm 0.02$ & $1.25 \pm 0.05$ & \\
\hline
\end{tabular}

characterizes these quasars is an artifact of the different focal-plane diaphragms used in making the infrared and visible observations and reflects the presence of a host galaxy associated with the quasar.

Although the exact separation into the two categories is sometimes ambiguous and depends on the redshift of the quasar, on the quality of the observations, and on the degree to which fits are accepted, all but one of the 105 observed continua, that of PG $2302+029$, are consistent with their belonging to one of these categories.

\section{a) “3000 A Bump" Amplitude}

It is well established that a " $3000 \AA$ bump" is present in the continua of many quasars (NOBM; Richstone and Schmidt 1980; Oke, Shields, and Korycansky 1984 and references therein). Since this feature is potentially present to some extent in all the continua, it was desirable to estimate its. strength in each of the quasars in the sample in order to delineate the underlying continuum.

An estimate of the contribution of the " $3000 \AA$ bump" to each quasar's continuum energy distribution requires an assumption about the shape of the bump as well as an assumption of the underlying continuum. For the present study, the observed flux densities were assumed to consist of a power-law continuum underlying a bump of a specified shape. WNW have presented observations from which they conclude that the " $3000 \AA$ bump" is made up of multiple lines of hydrogen and iron. On the basis of these observations, the shape of the "3000 A bump" was taken to be made up of two peaks: a peak, made up of $\mathrm{Fe}$ II lines, covering the range $\sim 14.99<$ $\log \left[\nu_{0}(\mathrm{~Hz})\right]<\sim 15.12$ plus a peak, made up of Balmer emission lines and a continuum, ranging from $\sim 14.90<$ $\log \left[\nu_{0}(\mathrm{~Hz})\right]<\sim 15.02$. The shapes of the assumed peaks, smoothed and averaged from seven model curves given by WNW, are shown in Figure 2. 


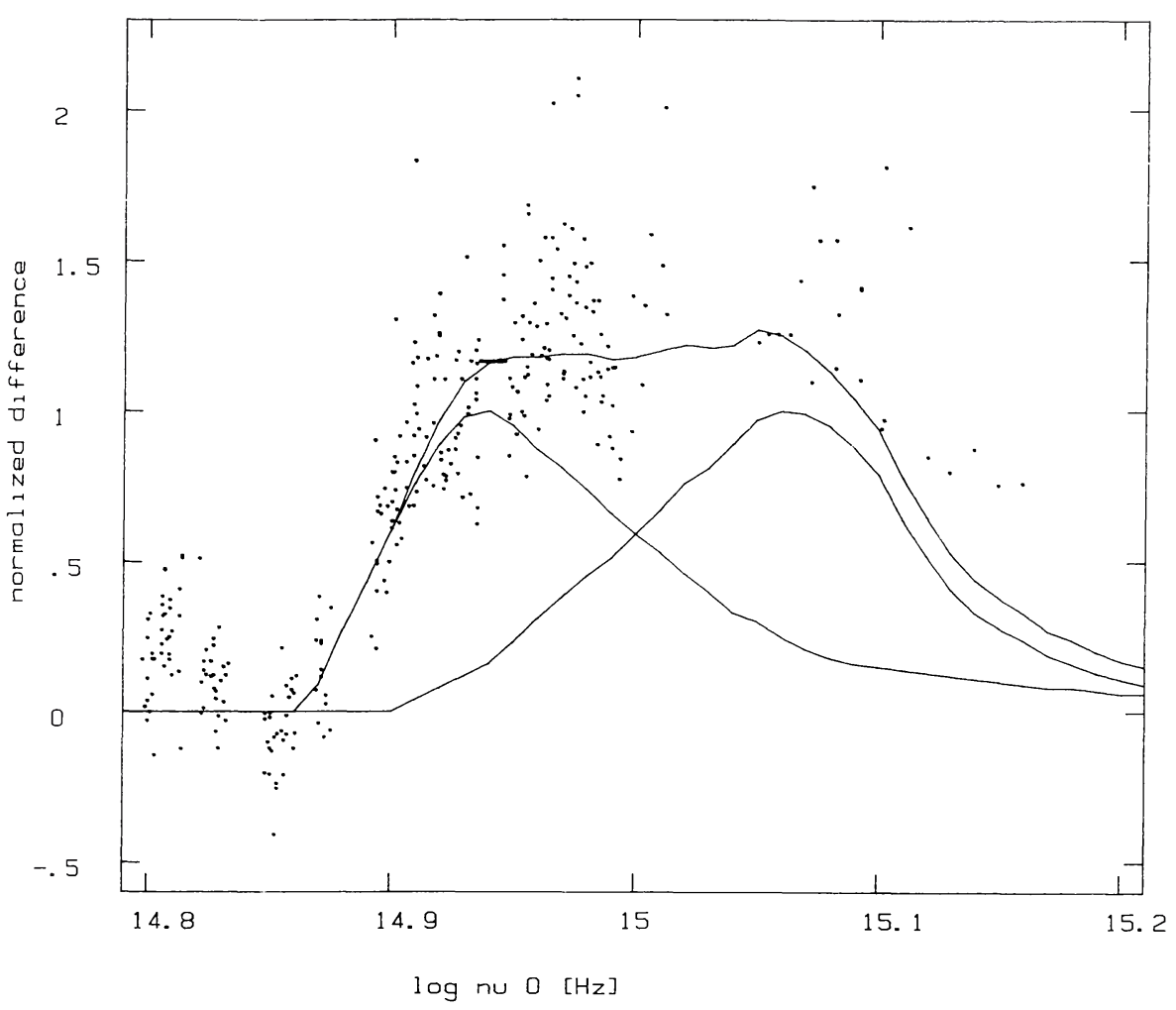

FIG. 2.-Differences between the observed flux densities and the assumed power-law continua are shown for frequencies near the " $3000 \AA \AA$ bump." The differences have been normalized either at $\log \left[\nu_{0}(\mathrm{~Hz})\right]=14.94$ or at $\log \left[\nu_{0}(\mathrm{~Hz})\right]=15.06$. The differences for 21 quasars are displayed for $\log \left[\nu_{0}(\mathrm{~Hz})\right]<15$, and those for three quasars for $\log \left[\nu_{0}(\mathrm{~Hz})\right]>15$. The solid line shows the iron and Balmer peaks from WNW and their sum assuming equal maximum amplitudes. Several of the observations have been adjusted for the known unequal amplitudes of the iron and Balmer peaks; see text.

The underlying continuum was evaluated by first obtaining a power-law fit to the observations at frequencies outside the range where the bump was prominent. The amplitudes of the iron and Balmer peaks were then found by fitting the difference between the observed flux densities and the power-law continuum with the shapes given by WNW but with adjustable amplitudes. The parameters of the power-law slope were then reevaluated in an iterative process using the flux densities at all the frequencies, including those in the vicinity of the bump, but with the contributions of the peaks, as initially derived, removed. For those quasars which could be fitted by two power-law slopes, only measurements at frequencies higher than the "break" frequency between the regimes of the two slopes were used. For those quasars where a clear discontinuity between the multichannel and infrared observations was evident, only observations obtained with the multichannel spectrometer were used. For 13 quasars, the calculations resulted in negative estimates of either or both of the iron and Balmer amplitudes. For the subsequent fittings, these quasars were assumed to have none of that peak present.

The maximum amplitudes of the peaks making up the bumps, measured relative to the underlying power-law continua, ranged up to 0.2 for the Balmer peak and up to 0.6 for the iron peak. The median of the maximum amplitudes of the Balmer peak relative to the power-law continuum at $\log [\nu(\mathrm{Hz})]=14.94$ is 0.14 , while the median of the maximum amplitudes of the iron peak relative to the power-law continuum at $\log \left[\nu_{0}(\mathrm{~Hz})\right]=15.06$ is 0.24 . Figure 3 shows that there is no marked dependence on the luminosity of the quasar of the amplitude of the Balmer peak, the peak with the most observations, relative to the assumed power-law continuum. The determination of the luminosities of the sample is described below.

For quasars with redshifts $z$ in the range $0.42<z<1.39$, it is possible in principle to determine the amplitudes of both the iron and Balmer peaks. Unfortunately, the background continua outside the wavelength range of the two peaks were often very poorly defined, and good determinations of the relative amplitudes of the iron and Balmer peaks were achieved for only 11 quasars out of the 22 in the appropriate redshift range. For these 11, the ratio of the maximum amplitude of the iron peak to that of the Balmer peak varied from 0.1 to 1.5 , with a mean of 0.8 and a median value of 1.0. In comparison, the model fits of WNW to observations of seven quasars show ratios of the maximum amplitudes that range from 1.0 to 4.1 with a median of 2.1. This difference is presumably due to selection biases.

For quasars with redshifts outside the range $0.42<z<1.39$, the amplitude of either the Balmer or the iron peak could not be determined from the observations. As evident from Figure 2 , however, the peaks overlap in frequency, so that the determination of either always depends, although not strongly, on the assumed amplitude of the other. When the frequency range was too small to define both peaks, equal amplitudes for the iron and Balmer peaks were assumed in order to estimate the strength of the peak in the observed range. 


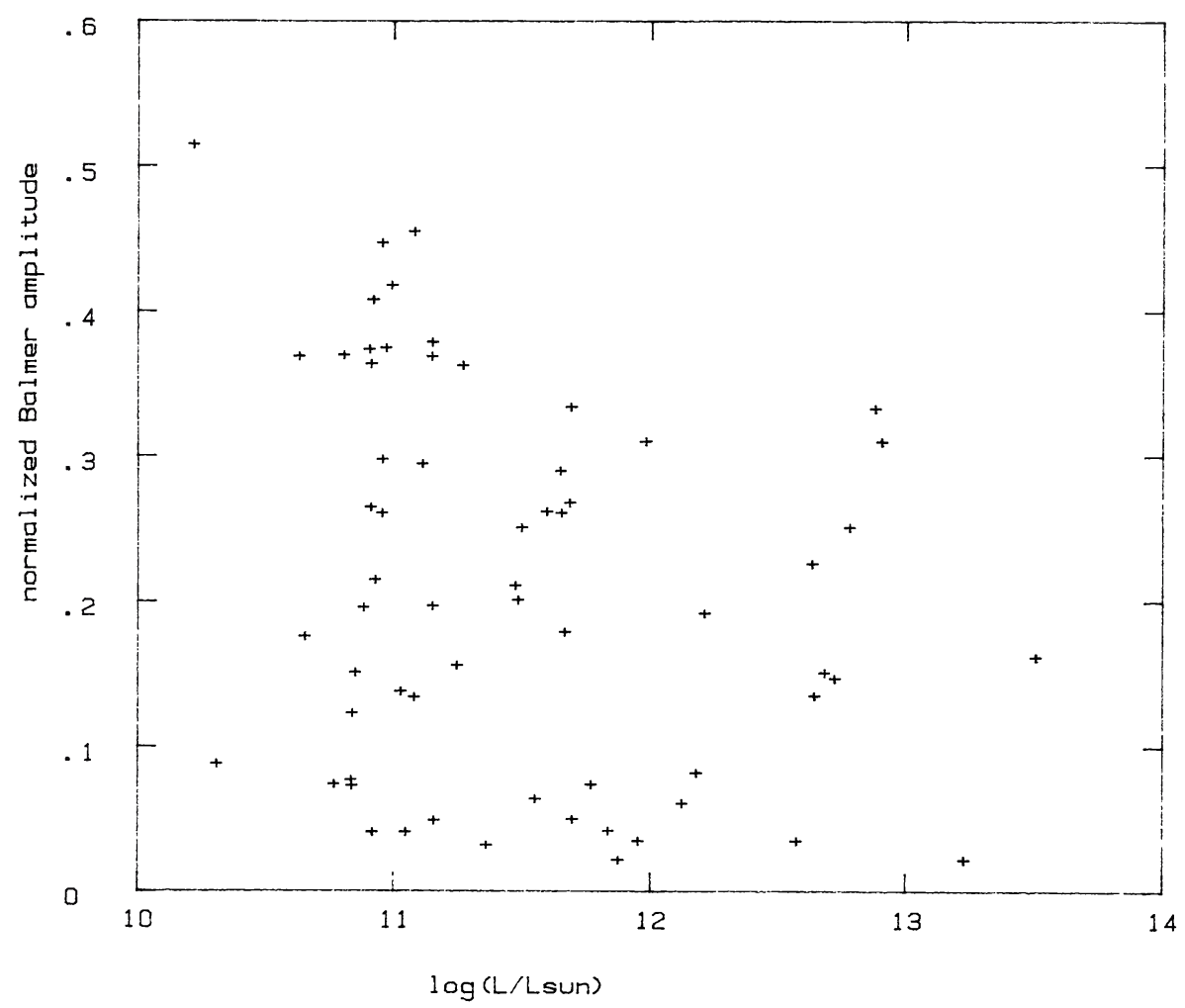

FIG. 3. - The maximum amplitude of the Balmer peak relative to the flux density obtained for the power-law fit to the continuum at $\log \left[\nu_{0}(\mathrm{~Hz})\right]=14.94$ is shown as a function of the luminosity at $\log \left[\nu_{0}(\mathrm{~Hz})\right]=14.5$.

Although the main purpose in evaluating the "3000 $\AA$ bump" amplitude was to remove its effects in the subsequent fitting procedures, it is informative to examine the derived shapes of the peaks, if only to check on the validity of the model. Figure 2 includes the differences between the observations and the assumed power-law continua for quasars with maximum amplitudes of the Balmer or iron peaks greater than 0.3 relative to the power-law continuum. Only observations with less than 5\% assigned uncertainty are included; these restrictions mean that only 21 quasar continua represent frequencies with $\log \left[\nu_{0}(\mathrm{~Hz})\right]<15$ and only three represent frequencies with $\log \left[\nu_{0}(\mathrm{~Hz})\right]>15$. The flux density differences for frequencies $\log \left[\nu_{0}(\mathrm{~Hz})\right]<15$ have been normalized at $\log \left[\nu_{0}(\mathrm{~Hz})\right]=14.94$, the frequency of. the maximum for the Balmer peak, while those with $\log \left[\nu_{0}(\mathrm{~Hz})\right]>15$ have been normalized at $\log \left[\nu_{0}(\mathrm{~Hz})\right]=15.06$, the frequency of the maximum for the iron peak. The smoothed models, as derived from WNW, of the iron peak, the Balmer peak, and their sum are also shown for the case when the two peaks are of equal amplitude. In order to facilitate comparison between the observations and the models, the differences for four of the quasars representing the Balmer peak have been adjusted to account for the presence of an iron peak with unequal amplitude, and two of the three continua at $\log \left[\nu_{0}(\mathrm{~Hz})\right]>15$ have been adjusted for the presence of a Balmer peak of unequal amplitude.

The resultant differences displayed in Figure 2 are seen to be in reasonable agreement with the sum of the smoothed WNW models. The iron peak is poorly defined not only because of the small number of quasars sampled, but also because the Balmer peak makes a significant contribution throughout the frequency range of the iron peak. It should be emphasized that the agreement between the observations and the shape of the models has in no way been forced by the fitting procedure. The only way the fitting procedure has entered is through the determination of the maximum amplitudes of the peaks and through the removal of the adjacent peak for those few cases where the two peaks were derived to be of unequal amplitude.

\section{b) Power-Law Fits}

The physical validity of characterizing the continua of quasars by power laws clearly depends on the range of wavelengths observed as well as on the intrinsic nature of the continuum. The " 3000 A bump," for instance, could, if improperly assessed, presumably mask any underlying power-law continuum. Likewise, thermal radiation which does not resemble a power law might make a significant contribution to the continuum in the infrared wavelengths. Nonetheless, it is informative to investigate simple power-law fits to the observations in order to delineate statistical trends.

Least-squares fits to the continua, with the " $3000 \AA$ A bump" removed as discussed above, are included in Figure 1. The parameters of the fits are given in Table 5. When two power laws were required, a "break" frequency between the two slopes was determined from visual inspection of the data, and the observations above and below that frequency were fitted independently to power laws by minimizing the least-squares differences from the observations. The validity of the initial 
TABLE 5

POWER-LAW SLOPES AND LuMINOSITIES

\begin{tabular}{|c|c|c|c|c|c|c|c|c|}
\hline \multirow[t]{2}{*}{ Object } & \multicolumn{3}{|c|}{ Low Frequency } & High & h Frequency & & $\log (L / L O)$ & $\overline{\text { notes }}$ \\
\hline & $\alpha$ & $\log \left[[\mathrm{mJy}]^{*}\right.$ & $x^{2+}$ & $\alpha$ & $\log \mathrm{I}[\mathrm{mJy}]^{*}$ & $x^{2+}$ & & \\
\hline $0002+051$ & - & - & - & -0.04 & 0.23 & 1.6 & 13.18 & 1 \\
\hline $0003+158$ & -0.12 & 0.41 & 2.6 & -0.12 & 0.41 & 2.6 & 11.98 & 2 \\
\hline $0007+106$ & -1.79 & 0.78 & 2.9 & -0.72 & 0.86 & 6.8 & 10.97 & \\
\hline $0026+129$ & -1.20 & 0.55 & 0.6 & -0.48 & 0.70 & 2.9 & 11.22 & 3 \\
\hline $0043+039$ & - & - & - & -0.28 & 0.27 & 4.4 & 11.70 & 4 \\
\hline $0044+030$ & -0.93 & 0.14 & 0.4 & -0.55 & 0.25 & 5.2 & 12.13 & 3 \\
\hline $0049+171$ & -0.83 & 0.40 & 6.4 & -0.83 & 0.40 & 6.4 & 10.22 & 2 \\
\hline $0052+251$ & -1.17 & 0.54 & 3.2 & -0.32 & 0.55 & 4.1 & 11.15 & \\
\hline $0117+213$ & -1.87 & 0.16 & - & -0.17 & 0.28 & 3.3 & 13.00 & \\
\hline $0157+001$ & -1.51 & 0.50 & 1.0 & -0.49 & 0.51 & 3.1 & 11.16 & \\
\hline $0 B 04+761$ & -1.60 & 0.82 & 0.4 & -0.10 & 0.87 & 8. 8 & 11.08 & \\
\hline $0832+251$ & -1.57 & 0.37 & 0.6 & +0.21 & 0.29 & 2.7 & 11.63 & \\
\hline $0838+770$ & -1.73 & 0.22 & 0.3 & -0.65 & 0.46 & 2.6 & 10.91 & 3 \\
\hline $0844+349$ & -0.82 & 0.84 & 0.3 & -0.87 & 0.94 & 3.3 & 10.65 & 3 \\
\hline $0906+484$ & -1.23 & 0.22 & 0.0 & -0.94 & 0.40 & 3.0 & 10.75 & 3 \\
\hline $0923+201$ & -1.71 & 0.37 & 0.5 & +0.10 & 0.25 & 4.2 & 11.15 & \\
\hline $0931+437$ & -1.70 & 0.10 & 3.2 & -0.11 & 0.06 & 3.9 & 11.69 & \\
\hline $0935+417$ & - & - & - & -0.16 & 0.21 & 2.7 & 13.20 & 1 \\
\hline $0946+301$ & - & - & - & -0.22 & 0.26 & 1.6 & 12.78 & $i$ \\
\hline $0947+396$ & -1.88 & -0.02 & 0.1 & -0.61 & 0.18 & 2.9 & 11.04 & 3 \\
\hline $0953+414$ & -1.43 & 0.49 & 1.7 & -0.02 & 0.50 & 2.5 & 11.49 & \\
\hline $1001+051$ & -1.59 & 0.28 & 0.8 & +0.10 & 0.14 & 3.4 & 10.92 & \\
\hline $1004+130$ & -1.16 & 0.45 & - & -0.15 & 0.63 & 1.9 & 11.63 & \\
\hline $1008+133$ & - & & - & +0.13 & 0.08 & 20 & 12.64 & 1 \\
\hline $1011-040$ & -0.95 & 0.63 & 0.4 & -1.04 & 0.82 & 2.6 & 10.36 & 3 \\
\hline $1012+008$ & -1.05 & 0.38 & 3.8 & -0.57 & 0.48 & 2.5 & 11.23 & 3 \\
\hline $1048-090$ & -1.16 & 0.17 & - & -0.16 & 027 & 46 & 1160 & \\
\hline $1048+342$ & -0.81 & 0.25 & 1.8 & -0.53 & 026 & 2.0 & 1093 & \\
\hline $1049-005$ & -1.95 & 0.26 & - & -0.23 & 031 & 1.5 & 11.67 & \\
\hline $1100+772$ & -0.96 & 0.41 & 0.6 & -0.11 & 0.37 & 6.3 & 11.65 & \\
\hline $1103-006$ & -1.07 & 0.17 & 0.6 & -0.22 & 0.14 & 2.7 & 11.69 & \\
\hline $1112+431$ & -1.92 & -0.37 & 4.6 & -0.15 & -0.44 & 2.2 & 10.84 & \\
\hline $1114+445$ & -1.25 & 0.49 & 0.7 & -0.67 & 0.43 & 2.2 & 11.03 & \\
\hline $1115+080$ & - & - & - & -0.15 & 0.27 & 4.0 & 13.12 & 1 \\
\hline $1115+407$ & -1.21 & 0.20 & 0.1 & -0.66 & 0.26 & 1.8 & 10.86 & 3 \\
\hline $1116+215$ & -1.70 & 0.78 & 1.1 & -0.14 & 0.76 & 3.5 & 11.50 & \\
\hline $1119+120$ & -0.95 & 0.73 & 1.1 & -1.11 & 0.89 & 2.5 & 10.31 & 3 \\
\hline $1121+422$ & -1.41 & 0.11 & 0.1 & -0.41 & 0.09 & 2.4 & 11.08 & \\
\hline $1126-041$ & -1.41 & 0.87 & 0.2 & -0.85 & 0.90 & 5.6 & 10.65 & \\
\hline $1138+040$ & - & - & - & -0.07 & -0.20 & 1.7 & 12.73 & 1 \\
\hline $1148+549$ & - & - & 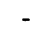 & -0.33 & 0.43 & 5.2 & 12.72 & 1 \\
\hline $1151+117$ & -1.45 & 0.18 & 0.1 & -0.49 & 0.24 & 1.0 & 10.95 & \\
\hline $1202+281$ & -2.01 & 0.25 & 0.3 & -0.96 & 0.26 & 2.6 & 10.92 & \\
\hline $1206+459$ & - & 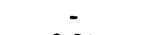 & - & -0.41 & 0.43 & 2.6 & 12.90 & 1 \\
\hline $1211+143$ & -1.37 & 0.91 & 0.1 & -0.26 & 1.08 & 3.3 & 11.14 & 3 \\
\hline $1216+069$ & -0.87 & 0.50 & 1.1 & -0.64 & 0.62 & 8.0 & 11.92 & 3 \\
\hline $1222+228$ & 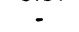 & & . & -0.50 & 0.44 & 2.0 & 13.45 & 1 \\
\hline $1226+023$ & -1.34 & 1.56 & 2.1 & -0.11 & 1.47 & 2.1 & 12.18 & \\
\hline $1229+204$ & -1.23 & 0.70 & 0.1 & -0.90 & 0.86 & 3.7 & 10.68 & 3 \\
\hline $1241+176$ & - & - & - & -0.71 & 0.67 & 0.7 & 13.23 & $\underline{1}$ \\
\hline $1244+026$ & -0.97 & 0.39 & 0.2 & -1.05 & 0.58 & 1.9 & 9.95 & 3 \\
\hline $1247+267$ & - & - & - & -0.32 & 0.50 & 2.9 & 13.51 & 1 \\
\hline $1248+401$ & - & - & - & -0.50 & 0.33 & 3.4 & 12.68 & $i$ \\
\hline $1254+047$ & - & - & - & -0.19 & 0.22 & 1.1 & 12.57 & 4 \\
\hline $1259+593$ & -1.25 & 0.31 & - & -0.00 & 034 & 3.0 & 11.95 & \\
\hline $1302-102$ & -1.13 & 0.49 & - & +0.28 & 0.61 & 0.8 & 11.77 & \\
\hline $1307+085$ & -0.95 & 0.49 & 0.5 & -0.29 & 0.51 & 2.4 & 11.11 & \\
\hline $1309+355$ & -0.97 & 0.45 & 2.0 & -0.80 & 0.54 & 1.2 & 11.29 & 3 \\
\hline $1310-108$ & -0.76 & 0.54 & 1.1 & -1.06 & 0.73 & 3.9 & 9.83 & 3 \\
\hline $1329+412$ & $\because$ & - & - & -0.14 & -0.28 & 4.2 & 12.68 & 1 \\
\hline $1333+176$ & -0.95 & 0.18 & 0.6 & -0.37 & 0.30 & 1.6 & 12.07 & 3 \\
\hline $1338+416$ & - & 80 & - & -0.10 & 0.11 & 1.8 & 12.63 & 1 \\
\hline $1351+236$ & -0.73 & 0.75 & 1.3 & -1.76 & 0.87 & 3.3 & 10.43 & \\
\hline $1351+640$ & -1.39 & 0.63 & 4.1 & -0.47 & 0.90 & 3.2 & 10.99 & 3 \\
\hline $1352+183$ & -1.45 & 0.26 & 3.3 & -0.34 & 0.34 & 2.6 & 10.95 & \\
\hline $1352+011$ & -0.79 & 0.20 & 0.3 & -0.43 & 0.42 & 1.7 & 12.85 & 3 \\
\hline $1354+213$ & - & - & - & -0.70 & -0.04 & 1.3 & 11.15 & 4 \\
\hline $1402+261$ & -1.92 & 0.40 & 1.6 & +0.20 & 0.31 & 2.6 & 11.05 & \\
\hline $1404+226$ & -0.93 & 0.18 & 0.1 & -0.56 & 0.32 & 2.2 & 10.52 & 3 \\
\hline $1407+265$ & - & - & - & -0.18 & 0.32 & 3.2 & 12.59 & 1 \\
\hline $1411+442$ & -1.83 & 0.63 & 0.6 & -0.70 & 0.73 & 3.3 & 10.83 & \\
\hline $1415+451$ & -1.43 & 0.50 & 0.2 & -0.99 & 0.51 & 3.3 & 10.84 & \\
\hline $1416-129$ & -0.92 & 0.43 & 5.6 & +0.32 & 0.55 & 2.0 & 10.99 & \\
\hline $1426+015$ & -1.19 & 0.80 & 1.4 & -0.31 & 0.88 & 4.8 & 10.95 & \\
\hline $1427+480$ & -1.15 & -0.04 & 0.8 & -0.35 & -0.01 & 2.0 & 10.81 & \\
\hline $1435-067$ & -1.61 & 0.29 & - & -0.00 & 0.47 & 3.0 & 10.90 & \\
\hline $1440+356$ & -1.31 & 0.90 & 4.9 & -0.86 & 0.86 & 3.4 & 10.88 & \\
\hline $1444+407$ & $\begin{array}{r}1.62 \\
-1.65\end{array}$ & 0.27 & 1.7 & +0.03 & 0.19 & 4.0 & 11.36 & \\
\hline $1448+273$ & -0.80 & 0.76 & 0.8 & -0.75 & 0.89 & 2.4 & 10.72 & 3 \\
\hline $1501+106$ & -1.03 & 0.99 & 0.1 & -0.32 & 1.11 & 4.5 & 10.42 & 3 \\
\hline $1512+370$ & -1.14 & 0.16 & 0.4 & -0.36 & 0.26 & 2.2 & $\begin{array}{l}11.65 \\
\text { 1. }\end{array}$ & \\
\hline $1519+226$ & -1.89 & 0.36 & 2.9 & -0.50 & 0.33 & 3.4 & 10.85 & \\
\hline $1522+101$ & -1.78 & 0.28 & 0.1 & +0.25 & 0.22 & 1.6 & 12.88 & \\
\hline
\end{tabular}


TABLE 5-Continued

\begin{tabular}{|c|c|c|c|c|c|c|c|c|}
\hline \multirow[t]{2}{*}{ Object } & \multicolumn{3}{|c|}{ Low Frequency } & \multicolumn{3}{|c|}{ High Frequency } & \multirow[t]{2}{*}{$\log (L / L)$} & \multirow[t]{2}{*}{ notes } \\
\hline & $\alpha$ & $\log 1[\mathrm{mJy}]^{*}$ & $x^{2+}$ & $\alpha$ & $\log \left[[\mathrm{mJy}]^{*}\right.$ & $x^{2+}$ & & \\
\hline $\begin{array}{l}1534+580 \\
1535+547 \\
1543+489 \\
1545+210 \\
1552+085 \\
1612+261 \\
1613+658 \\
1617+175 \\
1626+554 \\
1630+377 \\
1634+706 \\
1700+518 \\
1704+608 \\
1718+481 \\
2112+059 \\
2130+099 \\
2209+184 \\
2233+134 \\
2251+113 \\
2344+092 \\
2349-014\end{array}$ & $\begin{array}{l}-1.06 \\
-1.08 \\
-2.07 \\
-1.02 \\
-1.10 \\
-1.62 \\
-1.20 \\
-1.31 \\
-1.30 \\
- \\
-1.50 \\
-1.89 \\
-1.67 \\
-0.48 \\
-1.65 \\
-1.47 \\
-0.68 \\
-1.93 \\
-1.15 \\
- \\
-1.38\end{array}$ & $\begin{array}{c}0.75 \\
0.95 \\
0.09 \\
0.35 \\
0.31 \\
0.33 \\
0.56 \\
0.59 \\
0.26 \\
- \\
0.73 \\
0.61 \\
0.37 \\
0.86 \\
0.52 \\
0.79 \\
0.69 \\
-0.10 \\
0.57 \\
- \\
0.52 \\
\end{array}$ & $\begin{array}{c}0.3 \\
2.2 \\
- \\
0.0 \\
0.0 \\
0.0 \\
0.1 \\
1.5 \\
- \\
- \\
- \\
0.1 \\
0.8 \\
.5 \\
0.4 \\
2.9 \\
1.1 \\
0.1 \\
0.0 \\
- \\
0.9\end{array}$ & $\begin{array}{l}-1.21 \\
-1.08 \\
-0.16 \\
-0.13 \\
-0.37 \\
-0.87 \\
-1.35 \\
+0.13 \\
-0.69 \\
-0.21 \\
-0.11 \\
-0.62 \\
-0.43 \\
-0.48 \\
+0.41 \\
-0.34 \\
-0.92 \\
+0.23 \\
-0.10 \\
+0.10 \\
-0.57\end{array}$ & $\begin{array}{l}0.92 \\
0.95 \\
0.09 \\
0.38 \\
0.40 \\
0.50 \\
0.78 \\
0.49 \\
0.34 \\
0.26 \\
0.90 \\
0.75 \\
0.48 \\
0.86 \\
0.45 \\
0.85 \\
0.82 \\
-0.02 \\
0.47 \\
0.26 \\
0.57\end{array}$ & $\begin{array}{l}3.2 \\
2.2 \\
2.7 \\
1.4 \\
2.8 \\
8.7 \\
4.8 \\
6.1 \\
4.1 \\
1.8 \\
1.2 \\
3.3 \\
2.1 \\
1.5 \\
3.1 \\
5.0 \\
2.7 \\
1.6 \\
2.7 \\
2.5 \\
3.0\end{array}$ & $\begin{array}{c}9.90 \\
10.31 \\
11.55 \\
11.47 \\
10.76 \\
10.95 \\
11.00 \\
10.81 \\
10.80 \\
12.96 \\
13.51 \\
11.92 \\
11.87 \\
13.26 \\
12.12 \\
10.63 \\
10.58 \\
11.25 \\
11.84 \\
12.21 \\
11.27\end{array}$ & $\begin{array}{l}3 \\
2\end{array}$ \\
\hline
\end{tabular}

NoTes.-(1) Insufficient observations to determine whether two slopes with break near $1 \mu \mathrm{m}$ are required in fit. (2) Single slope assumed for both low and high frequencies. (3) Discrepant optical and infrared observations: high-frequency spectral index derived from multichannel observations, and low-frequency spectral index derived from infrared observations. (4) Observations require two slopes, but are insufficient to determine spectral index for low frequencies.

${ }^{*} I=$ power-law intercept at $\log \left[\nu_{0}(\mathrm{~Hz})\right]=14.5$

${ }^{\dagger}$ Reduced $\chi^{2}$.

choice of break frequency was checked after the fitting and iterated if necessary. This interactive approach was adopted because of the systematic difference in the uncertainties assigned to the visual and infrared observations, coupled with the fact that the break between the two slopes generally fell near the transition between the two observing techniques.

The continua of 20 of the quasars can be represented, over the observed frequency range, by a single power law plus the "3000 ̊ bump." Sixteen of these 20 quasars have such high redshifts that two slopes intersecting at $\sim 1 \mu \mathrm{m}$ could not be determined. Only four quasars (PG $0003+158$, PG 0049+171, PG $1535+547$, and PG $1718+481$ ) of the 20 have sufficiently low redshifts that the observations covered a broad enough range of rest wavelengths to define, in principle, two slopes intersecting at $\sim 1 \mu \mathrm{m}$. For four quasars (PG 0043+039, PG $1254+047$, PG 1354+213, PG 2344+092) of the 20, the "break" frequency resulting from the fitting procedure was sufficiently low that no low-frequency slope could be determined, although the lowest frequency observation available indicated that a continuation of the high-frequency power law was inappropriate.

Since an inspection of the continua showed that the very low frequency observations often clearly differed from a power-law extrapolation of the neighboring points, observations with $\log \left[\nu_{0}(\mathrm{~Hz})\right]<13.8$ were arbitrarily omitted from the fitting procedure; this is discussed below.

As indicated in Table 5, the reduced $\chi^{2}$ sums for the fits to the low-frequency observations are typically near unity. The reduced $\chi^{2}$ sums for the fits to the high-frequency observations are, however, typically 3 and range up to 8 , indicating either that the uncertainties are underestimated or that there is true structure such as the remains of the lines in the " 3000 $\AA$ bump" in the continuum that cannot be represented by power laws. This emphasizes the fact that the power laws should be considered only as statistical, not physical, representations of the continua. The reduced $\chi^{2}$ sum for PG $2302+029$, the one quasar not considered to be satisfactorily fitted by either one- or two-slope power laws, exceeded 100 . Strong curvature in the spectral energy distribution of that object continues into the ultraviolet; see Bechtold et al. (1987).

The distribution of the spectral indices $\alpha$ obtained with the best power-law fits to the observations is shown in Figure 4; $\alpha$ is defined such that $f_{\nu_{0}} \propto \nu_{0}^{\alpha}$. The quasars whose continua show evidence for a discontinuity between the multichannel and the infrared measurements have not been included in Figure 4, nor has the fit for the quasar PG 2302+029, which is considered unacceptable. The spectral indices of the four quasars that are best fitted by a single power law but whose range of rest frequencies extends well below $10^{14.5} \mathrm{~Hz}$ are included in the histograms of both the high- and low-frequency slopes and are marked in the figure. It is seen that all are among the flattest of the low-frequency indices and that one index, that of PG $0003+158$, is anomalously flat in comparison with the distribution of the other low-frequency spectral indices. The spectral indices of the power law describing the low-frequency observations have a median value of -1.4 and are thus significantly steeper than those describing the continua at higher frequencies, which have a median value of -0.2 . The full width at half-maximum of the distribution of low-frequency spectral indices is slightly wider than that for 


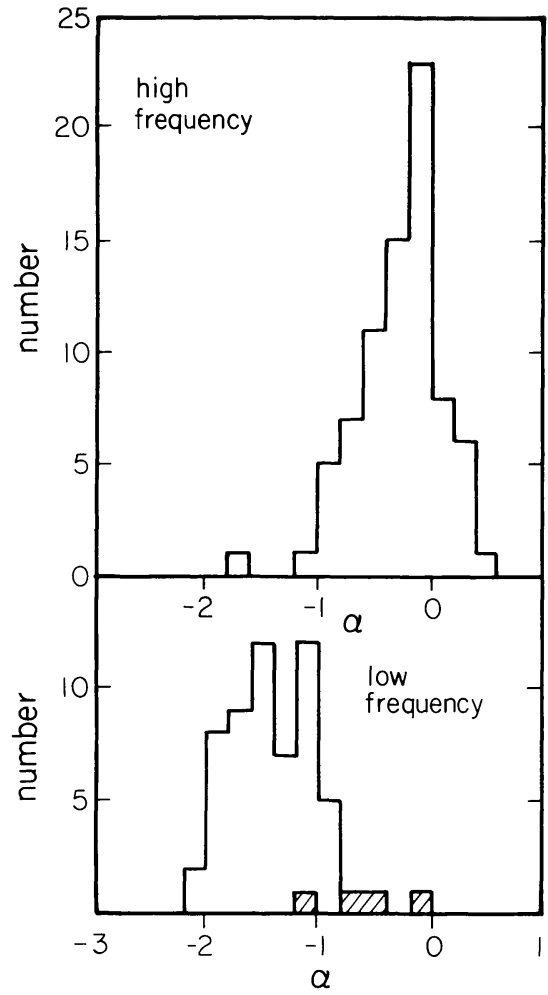

FIG. 4.-Histograms of the spectral indices for power-law fits are shown for fits above the "break" frequency (high-frequency indices) and those below the "break" frequency (low-frequency indices). The spectral indices of the continua for those four quasars whose continua are best fitted by a single power law and where the range of rest frequencies measured contains $\left.\log \left[\nu_{0} \mathrm{~Hz}\right)\right]=14.5$ are included in both histograms and are indicated in the lower panel by hatched boxes. For 20 quasars where the low-frequency spectral index could not be determined, only the high-frequency spectral indices are included. Spectral indices for quasars with discrepant multichannel and infrared measurements have been omitted.

the high frequencies -1.0 versus $0.8-$ and there is slight overlap between the two distributions. The possible correlation between the two slopes is shown in Figure 5 for that subset of 54 objects where there were sufficient observations to determine both; no strong correlation is seen.

The median spectral index of -0.2 for the high frequency power-law fit can be compared with the value of -0.5 found by Richstone and Schmidt (1980) for a sample of 85 objects, most of which were selected from radio surveys. Since these radio quasars have redshifts higher than those of the BQS quasars, and since Richstone and Schmidt did not explicitly remove the "3000 $\AA$ bump" in their power fitting, their derived slopes would be expected to be systematically different from the ones derived here. It is then interesting to note that the two distributions of spectral indices are very similar despite the selection for ultraviolet excess of the $\mathrm{BQS}$.

For those quasars where spectral indices in both the highand the low-frequency range were determined, it is possible to determine a "break" frequency between the high- and lowfrequency regimes. As shown in Figure 6, the range in this frequency is small, varying by less than a factor of 2 . The absolute value of the uncertainty in the determination of this frequency contributed by the uncertainty in the fits to the power laws ranges up to 0.13 , with a median value of 0.03 .

Fits with three power-law slopes were also made to the observations. Although these fits made improvements in a few selected cases, in general they were not significantly better than the fits with two or single power-law slopes.

\section{c) Luminosities}

For each quasar, the luminosity per logarithmic frequency interval, i.e., the product $\nu_{0} L_{\nu_{0}}$, where $L_{\nu_{0}}$ is the luminosity density per unit frequency interval, at $\sim 1 \mu \mathrm{m}\left(\log \left[\nu_{0}(\mathrm{~Hz})\right]=\right.$ 14.5), was taken as a measure of the near-infrared luminosity of the quasar. These luminosities are included in Table 5 . The Hubble constant was taken as $H_{0}=75 \mathrm{~km} \mathrm{~s}^{-1} \mathrm{Mpc}^{-1}$, and the deceleration parameter as $q_{0}=0.5$. For those 78 quasars where the multichannel and infrared observations formed a smooth continuum, the flux density at $\log \left[\nu_{0}(\mathrm{~Hz})\right]=14.5$ derived from the best power-law fit was used in this calculation. For those cases where the multichannel observations and infrared observations were discrepant, the higher flux density extrapolated to $\log \left[\nu_{0}(\mathrm{~Hz})\right]=14.5$ was used.

The luminosities of the quasars where the visual and infrared observations fit smoothly range from $1 \times 10^{10}$ to $3 \times 10^{13}$ $L_{\odot}$, with a median value of $4 \times 10^{11} L_{\odot}$. Since the mean spectral index at higher frequencies is greater than -1 , it should be noted that the peak luminosity of the quasar is not emitted in the near-infrared but is emitted at higher frequencies that those measured in these observations.

Figures 7 and 8 show that the spectral indices and the differences between the indices obtained for the high- and the low-frequency observations do not depend on the luminosity of the object. As noted above, the amplitude of the " $3000 \AA$ bump" is also not a function of the luminosity.

\section{d) Low-Frequency Observations}

It is clear from visual examination of Figure 1 that the observations made at $10.1 \mu \mathrm{m}$ in the observer's frame often are significantly different from a power-law fit to the rest of the observations. In Figure 9 a histogram is given of the differences between the observed flux densities and those predicted on the basis of the power-law fits at low frequencies. The differences have been normalized by the errors in the measurements, and only observations at rest frequencies $\nu_{0}<$ $10^{13.8} \mathrm{~Hz}$ are included. In addition two quasars have upper limits below the power-law extrapolation, one-PG 1202+ 281 - being significantly low. Although the median value of the differences shown in Figure 9 plus the limits, is only slightly above zero, it is clear that there are large asymmetric deviations in the sense that the observed value is less than that predicted on the basis of the other observations. As a measure of the asymmetry which is present, half of the observations which lie above the power-law extrapolation exceed the extrapolation by a factor between 1.25 and 1.9 , while half of those which lie below the extrapolation are less than the power-law extrapolation by a factor of between 2.1 and 3.5.

The significance of this pronounced asymmetry is not clear. The dispersion exhibited in Figure 9 may be due to variations 


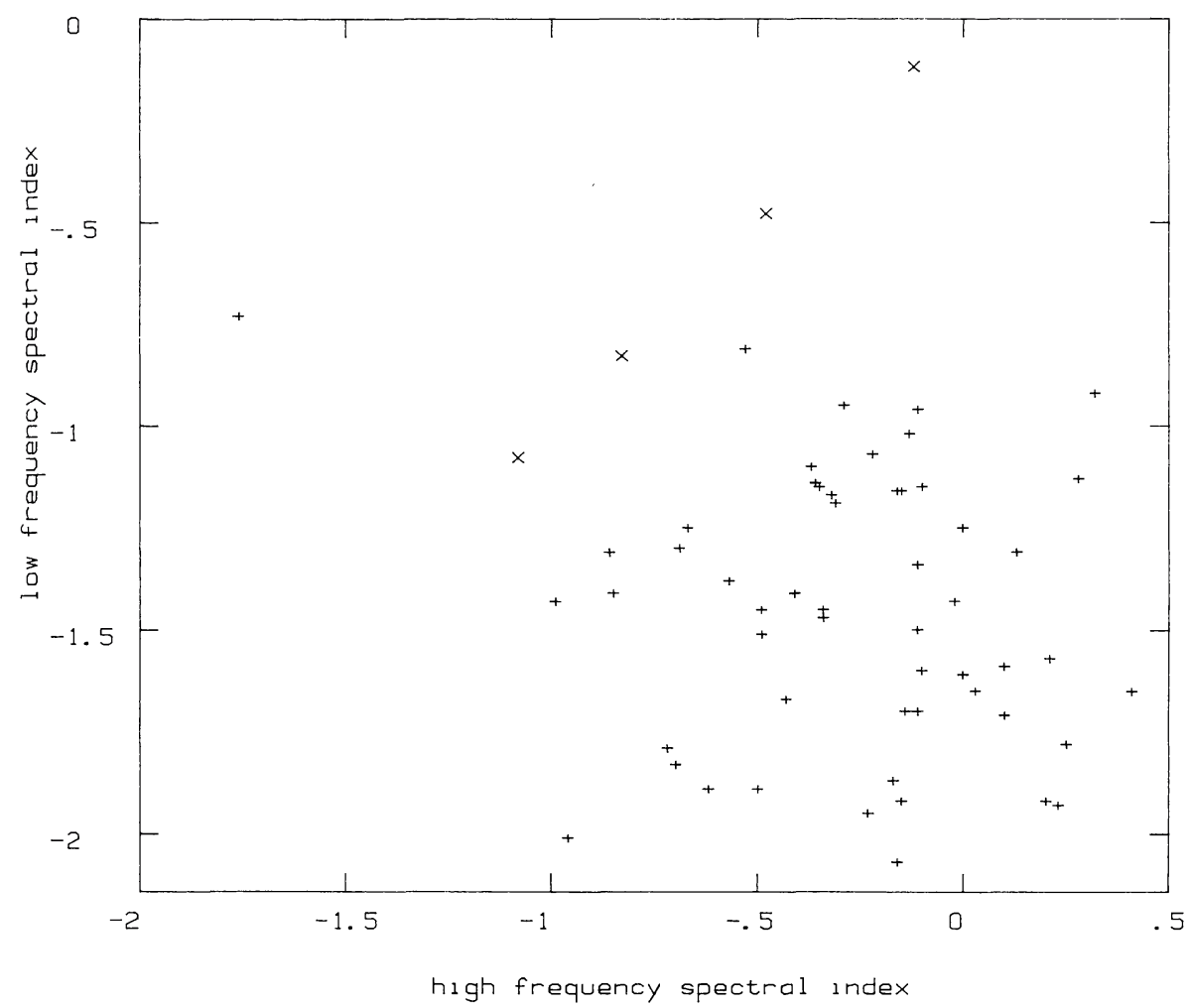

FIG. 5. - Spectral indices below the "break" frequency (low frequency) are shown as a function of those above the "break" frequency (high frequency) for those 54 quasars where both slopes can be determined. The spectral indices of the four quasars best fitted by a single power law are indicated with a cross $(\times)$. The spectral indices for quasars with discrepant multichannel and infrared measurements are excluded.

in the 10.1-2.2 $\mu \mathrm{m}$ color, but the systematic asymmetry is inconsistent with this hypothesis. In the canonical picture of the continuum emission of radio-loud quasars, the lowfrequency emission is attributed to an extension of the radio continuum emission from the inner regions of the quasars. The asymmetry in Figure 9 is consistent with the assumption that in some quasars the low-frequency continuum should not be represented by a power-law but rather is dominated by

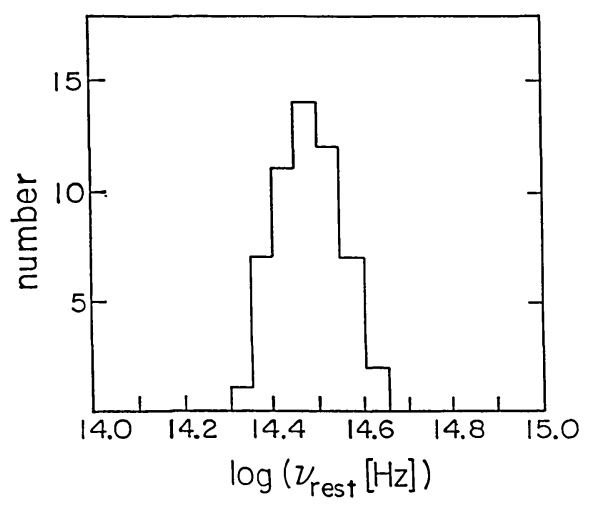

FIG. 6.-A histogram of the "break" frequency between the power-law fits to the low- and high-frequency observations is shown for 54 quasars whose continua could be fitted by two slopes. The spectral indices for quasars with discrepant multichannel and infrared measurements are omitted. thermal emission with a maximum at $\log \left[\nu_{0}(\mathrm{~Hz})\right] \sim 14$, i.e., temperatures $\sim 1000 \mathrm{~K}$. The data are insufficient to determine whether the peak is located near $3 \mu \mathrm{m}$, as suggested by NOBM, or at a longer wavelength. Edelson and Malkan (1986) have, in fact, found evidence for a peak in the continuum of several active galaxies at wavelengths around $5 \mu \mathrm{m}$. The small range in the "break" frequencies is also more consistent with the picture in which the break is produced by the superposition of a thermal component with a narrow range of maximum temperatures onto the underlying continuum components. The constraint of a narrowly regulated maximum dust temperature may be less restrictive than the requirement of a nearly constant ratio of disk to power-law emission.

\section{e) Continua Distorted by Quasar Host Galaxies}

A visual examination of the continua in Figure 1 indicates that there are at least 26 quasars for which the measurements with the multichannel spectrometer did not join smoothly with those in the infrared; these 26 are so indicated in Table 2. Checks on a sequence of standard stars show that the discontinuity is not an artifact of the absolute calibrations used in the two techniques.

Fifteen of the PG quasars were included in a search for infrared nebulosity around quasars (Neugebauer et al. 1985, hereafter, NMSE), thus allowing a comparison with the present observations. Of the 15 , seven were characterized in the 


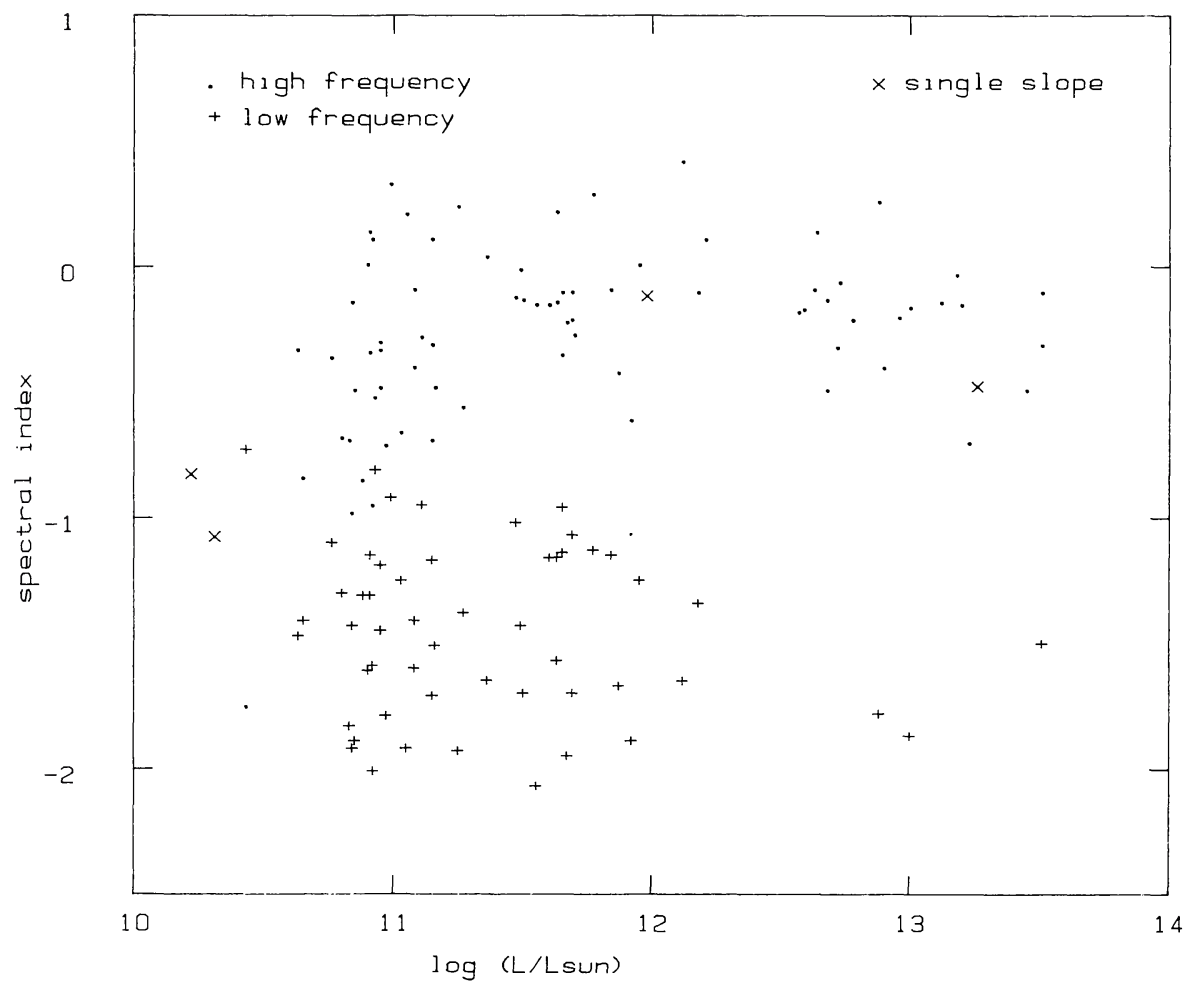

FIG. 7.-Spectral indices for power-law fits are plotted a a function of the quasar luminosity at $\log \left[\nu_{0}(\mathrm{~Hz})\right]=14.5$ for fits above the "break" frequency (high-frequency indices) and for fits below the "break" frequency (low-frequency indices). The spectral indices of the continua for those four quasars where the range of rest frequencies measured contains $\log \left[\nu_{0}(\mathrm{~Hz})\right]=14.5$ and whose continua are best fitted by a single power law are specially indicated. For 20 quasars where the low-frequency spectral index could not be determined, only the high-frequency spectral indices are included. Spectral indices for quasars with discrepant multichannel and infrared measurements have been omitted.

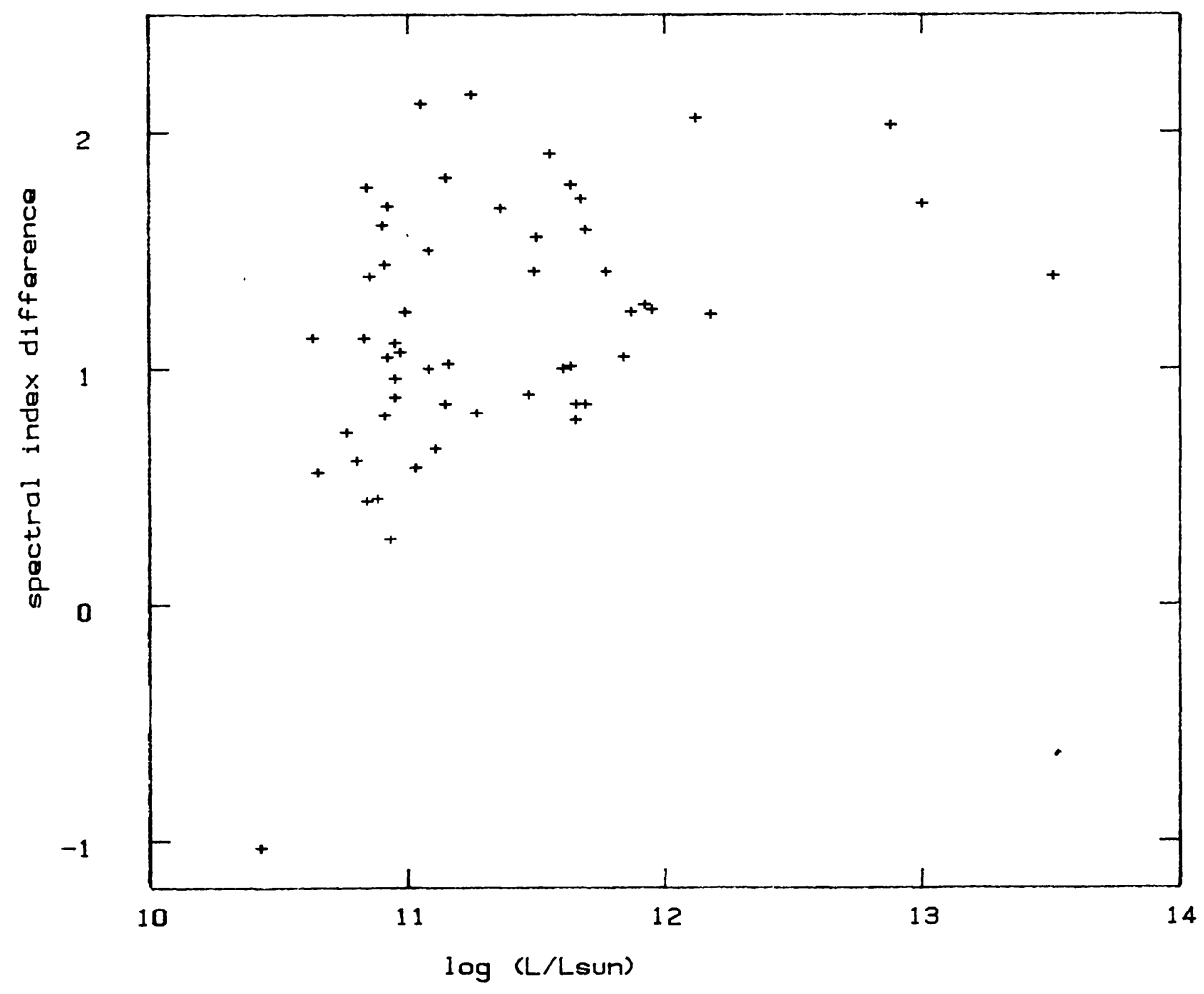

Fig. 8. - Differences between the spectral indices at low and high frequencies are shown as a function of the luminosity at $\log \left[\nu_{0}(\mathrm{~Hz})\right]=14.5$ for 54 quasars whose continua could be fitted by two slopes. The spectral indices for quasars with discrepant multichannel and infrared measurements are omitted. 


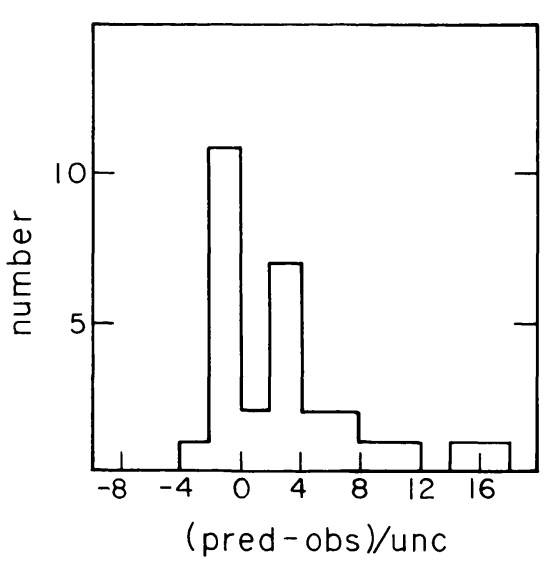

FIG. 9.-A histogram of the differences between the predicted flux densities based on power-law fits to higher frequency observations and those observed is shown for observations with $\log \left[\nu_{0}(\mathrm{~Hz})\right]<13.8$. The differences have been normalized by the individual uncertainties in the observed values; these uncertainties are typically $20 \%-30 \%$. In addition to the differences shown, three quasars have upper limits, of which two - those for PG $1202+281$ and PG $1307+085$ - are less than that predicted by the low-frequency power-law fit.

present study as having a discontinuity between the visual and infrared observations, and all of these seven exhibited evidence for infrared nebulosity in the observations of NMSE. Specifically, a measure of the nebulosity is provided by the ratio of the flux $F_{a}$ measured by NMSE in an annulus of inner diameter $5^{\prime \prime}$ and outer diameter $10^{\prime \prime}$ to the flux $F_{c}$ measured in a central $5^{\prime \prime}$ diameter diaphragm. Although there is a weak correlation between the discontinuity at $\sim 1 \mu \mathrm{m}$ and the ratio $F_{a} / F_{c}$, there are sufficient uncertainties in the extrapolations of the measurements that it is impossible to make a significant quantitative comparison between the discontinuity observed in Figure 1 and the nebulosity measured by NMSE. It is suggestive, however, that the seven quasars whose continua were associated with a discontinuity show a median ratio $F_{a} / F_{c} \sim 0.15$, while the eight which had no obvious discontinuity had a median ratio $F_{a} / F_{c} \sim 0.02$; the ratios for the former class ranged up to 0.39 , while none of the latter eight exceeded 0.09 .

Since the majority of the PG quasars have redshifts $z<0.3$, the transition between the multichannel observations and those with the infrared photometer often occurs near the "break" frequency, and it is possible qualitatively to associate the infrared observations with the low-frequency power-law slopes and the multichannel observations with the highfrequency power-law. The spectral indices derived from the separate observations are shown in Figure 10 for those 26 quasars designated as having significant discontinuities. It is seen that the range of indices derived from the infrared observations agrees qualitatively with that of the lowfrequency indices of those quasars with no discontinuity; if anything, the infrared observations produce flatter power laws. In contrast, the indices found from the observations made with the multichannel spectrometer are systematically steeper than the high-frequency indices in Figure 4. The nebulosity associated with quasars is presumably evidence of

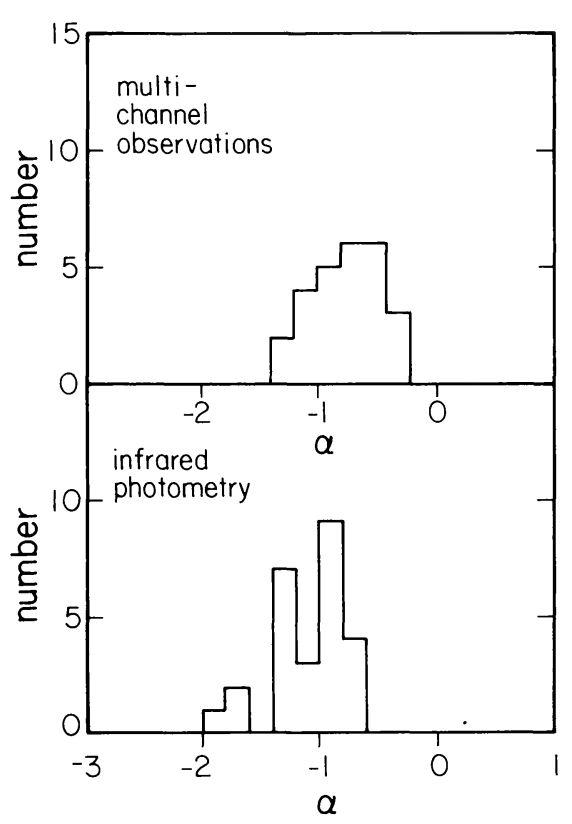

FIG. 10.-The spectral indices are shown as in Fig. 4, but for those quasars where the observations with the multichannel spectrometer and a $15^{\prime \prime}$ diameter diaphragm were inconsistent with those made using the infrared photometer and a 5".5 diameter diaphragm. The indices obtained from the observations with the infrared photometer should be compared with the "low"-frequency indices in Fig. 4, while the indices derived from the observations with the multichannel spectrometer should be compared with the "high"-frequency indices in Fig. 4.

host galaxies. Both of the effects just noted are consistent with an added galactic component with a maximum near $1.5 \mu \mathrm{m}$.

For those quasars where the visual and infrared observations are discordant, the luminositites ranged from $1 \times 10^{10}$ to $7 \times 10^{12} L_{\odot}$, with a median value of $7 \times 10^{10} L_{\odot}$. The difference between this median value and that $\left(4 \times 10^{11} L_{\odot}\right)$ for the other quasars presumably reflects the fact that less luminous quasars are selectively observed when they are closer. Specifically, it should be noted that the group of quasars with discontinuities in their energy distributions consists predominantly of the lower redshift quasars in the sample, again consistent with the nearby host galaxies which are spatially resolved. Of the 51 quasars in the entire sample with $z<0.2$, 21 show clear discontinuities. Of the 26 quasars whose observations show a discontinuity, all but one-PG $1352+011$ - have redshifts $z<1.0$.

Although no single piece of evidence is conclusive, there are thus a number of considerations which suggest that the discontinuities are artifacts of the different-sized diaphragms used in the visual and infrared observations. We thus conclude that, for the 26 sources with marked discontinuities, the observed red to near-infrared continua are not representative of the quasar per se, but contain a significant contribution from a surrounding galaxy.

\section{CONCLUSIONS}

In a study of nearly simultaneous visible spectrophotometry and near-infrared photometry of 105 quasars found in the optically selected PG survey, it is found that 
1. The continuum of each of the majority of the quasars can be fitted over the region $13.8<\log \left[\nu_{0}(\mathrm{~Hz})\right]<15$ with two simple power laws plus a “ $3000 \AA$ bump." The spectral indices at lower frequencies cluster around a median spectral index of -1.4 , while those at higher frequencies exhibit a median spectral index of -0.2 . At rest frequencies less than $10^{13.8} \mathrm{~Hz}$, the observations are systematically lower than an extrapolation of a power-law representation.

2. The transition frequency between the power-law slopes at low and high frequencies has a range of about one octave centered near $1 \mu \mathrm{m}$.

3. The power-law slopes do not depend critically on the luminosity of the quasars.

4. The observed " $3000 \AA$ bump" is consistent with components of iron and Balmer lines as proposed by WNW. The median maximum amplitudes of the iron and Balmer peaks are roughly equal. There is no strong correlation between the strength of the "bump" and the luminosity of the quasar.

5. A significant fraction of low-redshift quasars show evidence of nebulosity associated with the quasar in the difference between observations using a $6^{\prime \prime}$ diameter focal-plane diaphragm and observations with a $15^{\prime \prime}$ diameter focal-plane diaphragm.

A long-term project like this has taken effort by many people, especially at Palomar Observatory, and we thank them all, but particularly our night assistants J. Carrasco and G. Tuton. Much of the observing was done together with J. Elias, and we thank him for this and for many conversations. This work was supported by grants from the National Science Foundation.

\section{REFERENCES}

Bechtold, J., Czerny, B., Elvis, E., Fabbiano, G., and Green, R. 1987, Ap. $J .$, in press.

Burstein, D., and Heiles, C. 1984, Ap. J. Suppl., 54, 33.

Edelson, R., and Malkan, M. 1986, Ap. J., 308, 59.

Elias, J. H., Frogel, J. A., Matthews, K., and Neugebauer, G. 1982, A.J., 87, 1029.

Elvis, M., Green, R. F., Bechtold, J., Schmidt, M., Neugebauer, G.,

Soifer, B. T., Matthews, K., and Fabbiano, G. 1986, Ap. J., 310, 291.

Green, R. F., Schmidt, M., and Liebert, J. 1986, Ap. J. Suppl., 61, 305.

Neugebauer, G., Matthews, K., Soifer, B. T., and Elias, J. H. 1985, $A p$. $J .$, 298, 275 (NMSE).

Neugebauer, G., Oke, J. B., Becklin, E. E., and Matthews, K. 1979, Ap. $J .$, 230, 79 (NOBM).

Oke, J. B. 1969, Pub. A.S.P., 81, 11

Oke, J. B., and Gunn, J. 1983, Ap. J., 266, 713.

Oke, J. B., and Lauer, T. R. 1979, Ap. J., 230, 360.

Oke, J. B., Shields, G. A., and Korycansky, D. G. 1984, Ap. J., 277, 64.

Richstone, D. O., and Schmidt, M. 1980, Ap. J., 235, 361.

Rieke, G. H., and Lebofsky, M. J. 1985, Ap. J., 288, 618.

Schmidt, M., and Green, R. F. 1983, Ap. J., 269, 352.

Tokunaga, A. 1984, A.J., 89, 172.

van de Hulst, H. C. 1946, Rech. Astr. Obs. Utrecht, Vol. 11, Part 1.

Wampler, J. 1985, preprint.

Wills, B. J., Netzer, H., and Wills, D. 1985, Ap. J., 288, 94 (WNW).

J. Bennett, K. Matthews, G. Neugebauer, and B. T. Soifer: California Institute of Technology, George Downs Laboratory of Physics 320-47, Pasadena, CA 91125

R. Green: Kitt Peak National Observatory, National Optical Astronomy Observatories, 920 North Cherry, P.O. Box 26732, Tucson, AZ 85726

M. Schmidt: California Institute of Technology, Physics, Mathematics, and Astronomy 105-24, Pasadena, CA 91125 